\title{
Tomato SR/CAMTA transcription factors SISR1 and SISR3L negatively regulate disease resistance response and SISR1L positively modulates drought stress tolerance
}

Xiaohui Li, Lei Huang, Yafen Zhang, Zhigang Ouyang, Yongbo Hong, Huijuan Zhang, Dayong Li and Fengming Song*

\begin{abstract}
Background: The SR/CAMTA proteins represent a small family of transcription activators that play important roles in plant responses to biotic and abiotic stresses. Seven SISR/CAMTA genes were identified in tomato as tomato counterparts of SR/CAMTA; however, the involvement of SISRS/CAMTAs in biotic and abiotic stress responses is not clear. In this study, we performed functional analysis of the SISR/CAMTA family for their possible functions in defense response against pathogens and tolerance to drought stress.

Results: Expression of SISRs was induced with distinct patterns by Botrytis cinerea and Pseudomonas syringae pv. tomato (Pst) DC3000. Virus-induced gene silencing (VIGS)-based knockdown of either SISR1 or SISR3L in tomato resulted in enhanced resistance to $\mathrm{B}$. cinerea and Pst DC3000 and led to constitutive accumulation of $\mathrm{H}_{2} \mathrm{O}_{2}$, elevated expression of defense genes, marker genes for pathogen-associated molecular pattern-triggered immunity, and regulatory genes involved in the salicylic acid- and ethylene-mediated signaling pathways. Furthermore, the expression of SISR1L and SISR2L in detached leaves and whole plants was significantly induced by drought stress. Silencing of SISRIL led to decreased drought stress tolerance, accelerated water loss in leaves, reduced root biomass and attenuated expression of drought stress responsive genes in tomato. The SISR1 and SISR3L proteins were localized in the nucleus of plant cells when transiently expressed in Nicotiana benthamiana and had transcriptional activation activity in yeast.
\end{abstract}

Conclusions: VIGS-based functional analyses demonstrate that both SISR1 and SISR3L in the tomato SISR/CAMTA family are negative regulators of defense response against B. cinerea and Pst DC3000 while SISR1L is a positive regulator of drought stress tolerance in tomato.

Keywords: Tomato (Solanum lycopersicum), SR/CAMTA, Disease resistance response, Drought stress

\section{Background}

Plants are vulnerable to various biotic and abiotic stresses but have evolved to equip with sophisticated signaling networks to precisely regulate defense response to unfavorable stresses. Upon perception of environmental stress, a set of early signaling events including changes in the cytosolic free calcium $\left(\mathrm{Ca}^{2+}\right)$ concentration signatures (i.e. oscillations varying in cellular location, amplitude, duration or frequency) is often activated and integrated into different signaling pathways, which ultimately initiate transcriptional

\footnotetext{
* Correspondence: fmsong@zju.edu.cn

National Key Laboratory for Rice Biology, Institute of Biotechnology, Zhejiang University, Hangzhou 310058, China
}

(C) 2014 Li et al.; licensee BioMed Central Ltd. This is an Open Access article distributed under the terms of the Creative Commons Attribution License (http://creativecommons.org/licenses/by/4.0), which permits unrestricted use, distribution, and reproduction in any medium, provided the original work is properly credited. The Creative Commons Public Domain Dedication waiver (http://creativecommons.org/publicdomain/zero/1.0/) applies to the data made available in this article unless otherwise stated. reprogramming leading to expression of a large set of stress-responsive genes [1-3]. Extensive biochemical and genetic studies have demonstrated that cellular $\mathrm{Ca}^{2+}$ as a universal second messenger plays critical roles in regulating defense responses to diverse biotic and abiotic stresses [4-7].

Cellular $\mathrm{Ca}^{2+}$ changes can be sensed and interpreted by calcium-binding proteins (CaBPs) including calmodulin $(\mathrm{CaM})$, calcineurin B-like proteins and calcium-dependent protein kinases [8-10]. These $\mathrm{CaBPs}$ regulate cellular responses through two distinct pathways upon sensing different biotic and abiotic signals [8-10]. Firstly, CaBPs trigger rapid responses by direct binding to cytosolic 
target proteins and modulating their activity. Alternatively, CaBPs modulate indirect and relatively slow cellular responses by interacting with transcription factors to regulate gene expression. Recently, a class of CaM-binding transcription factors (CAMTA for CaM-binding transcription activator) has been identified in plants [11-14]. The CAMTA proteins, also called signal-responsive (SR) proteins, are present in all plant and animal species examined to date and are highly conserved in their protein structures. Typically, the SR/CAMTA proteins contain a CG-1 DNA-binding domain (binding to specific cis-elements in promoter regions of the target genes) at the N-terminus, a TIG domain (an immunoglobulin-like fold involved in nonspecific DNA binding), three ankyrin repeats (implicated in protein-protein interaction) and five putative CaM-binding motifs called as IQ motif [11-13]. Biochemical studies with the Arabidopsis AtSR1 and rice OsCBT proteins have identified the primary target of DNA ciselement for SR/CAMTA proteins as CGCG and CGTG motifs in promoter regions of the target genes [12,14].

The SR/CAMTA proteins represent a small family of transcription activators in plants. For instance, six genes encoding for SR/CAMTA proteins were identified in Arabidopsis [11]. The SR/CAMTA genes were shown to be responsive to multiple abiotic and biotic stresses including cold, wounding, drought and pathogen attack, as well as to stress-related hormones like ethylene, auxin, methyl jasmonate (MeJA) and salicylic acid (SA) [11,12,15-18]. Recent genetic studies with loss-of-function and gain-offunction mutants have shown that members of the SR/ CAMTA family play important roles in plant response to abiotic and biotic stresses. The Arabidopsis AtSR1 knockout mutant showed enhanced disease resistance against multiple pathogens with different infection styles including Pseudomonas syringae pv. tomato (Pst), Botrytis cinerea and Golovinomyces cichoracearum [19-21] but decreased resistance against insect herbivores [22,23]. By contrast, overexpression of AtSR1 conferred an increased susceptibility to Pst DC3000, B. cinerea and G. cichoracearum [21,24]. Similarly, the rice oscbt mutant exhibited significant resistance to blast fungal pathogen Magnaporthe grisea and leaf blight bacterial pathogen Xanthomonas oryzae pv. oryzae [17]. On the other hand, it was recently shown that the Arabidopsis AtSR1 and AtSR2 also play important roles in regulating tolerance to low temperature [25,26] and drought stress [27]. The function of SR/CAMTA proteins in plant biotic and abiotic stress response is achieved mainly through regulating expression of genes whose promoter regions contain the CGCG boxes [20,21,25,27].

Seven SlSRs/CAMTAs (hereafter referred to as SlSRs for convenience) genes were identified in tomato and were shown to be developmentally regulated during fruit development and ripening and induced by ethylene [28,29].
Further detailed analysis revealed that the SISR genes showed differential expression patterns in tomato fruit in response to low temperature, mechanical injury, infection of the necrotrophic fungal pathogen $B$. cinerea, and treatments with the signaling molecules SA and MeJA [30]. However, direct genetic evidence supporting the involvement of the tomato SlSRs in biotic and abiotic stress responses is still lacking. In the present study, we investigated the possible functions of SlSRs in disease resistance and drought stress tolerance using virus-induced gene silencing (VIGS) approach. Our VIGS-based functional analyses demonstrate that both SISR1 and SISR3L are negative regulators of defense response against $B$. cinerea and Pst DC3000 while SISR1L is a positive regulator of drought stress response.

\section{Results}

\section{Expression patterns of SISRs in response to pathogen infection}

To explore the possible functions of SlSRs in defense response against pathogen infection, we analyzed the expression patterns of SlSR genes in tomato plants after infection with different pathogens, $B$. cinerea, a necrotrophic fungal pathogen causing grey mold disease, and Pst DC3000, a (hemi) biotrophic bacterial pathogen causing bacterial leaf spot disease. In analysis of expression patterns of SISRs in response to infection of B. cinerea, leaf samples collected from the whole plant inoculation assays were used and the expression pattern of SILapA, a defense gene regulated by the JA/ET-mediated signaling pathway [31], was monitored to confirm the efficiency of the inoculation procedure. As shown in Figure 1, the expression level of SlLapA in B. cinerea-inoculated plants increased significantly, leading to 50 folds of increase at $24 \mathrm{hr}$ post inoculation (hpi) and $>300$ folds of increase at 48 hpi relative to those in the mock-inoculated plants, suggesting that our inoculation assays were appropriate for further analysis of the expression patterns of SlSRs in response to B. cinerea. Data from repeated qRT-PCR analyses revealed that the expression of almost all SlSRs was induced by infection of $B$. cinerea and the induced expression of SISRs was evident after 24 hpi and showed distinct patterns (Figure 1). The expression levels of SISR1, SISR1L, SISR2L and SISR3L were induced significantly by $B$. cinerea, leading to increases of $8,8,3$ and 15 folds at 24 hpi and of 8, 20, 7 and 90 folds at $48 \mathrm{hpi}$, respectively, over those in the mock-inoculated plants (Figure 1). The expression level of SlSR4 in the B. cinerea-inoculated plants showed 3 -fold increase at $12 \mathrm{hpi}$, peaked with 7 -fold of increase at $24 \mathrm{hpi}$ and then declined to the level in the mock-inoculated plants (Figure 1). By contrast, the expression levels of SISR2 and SISR3 in the B. cinerea-inoculated plants exhibited slight increases with less than 2 folds as compared with those in the mock-inoculated 


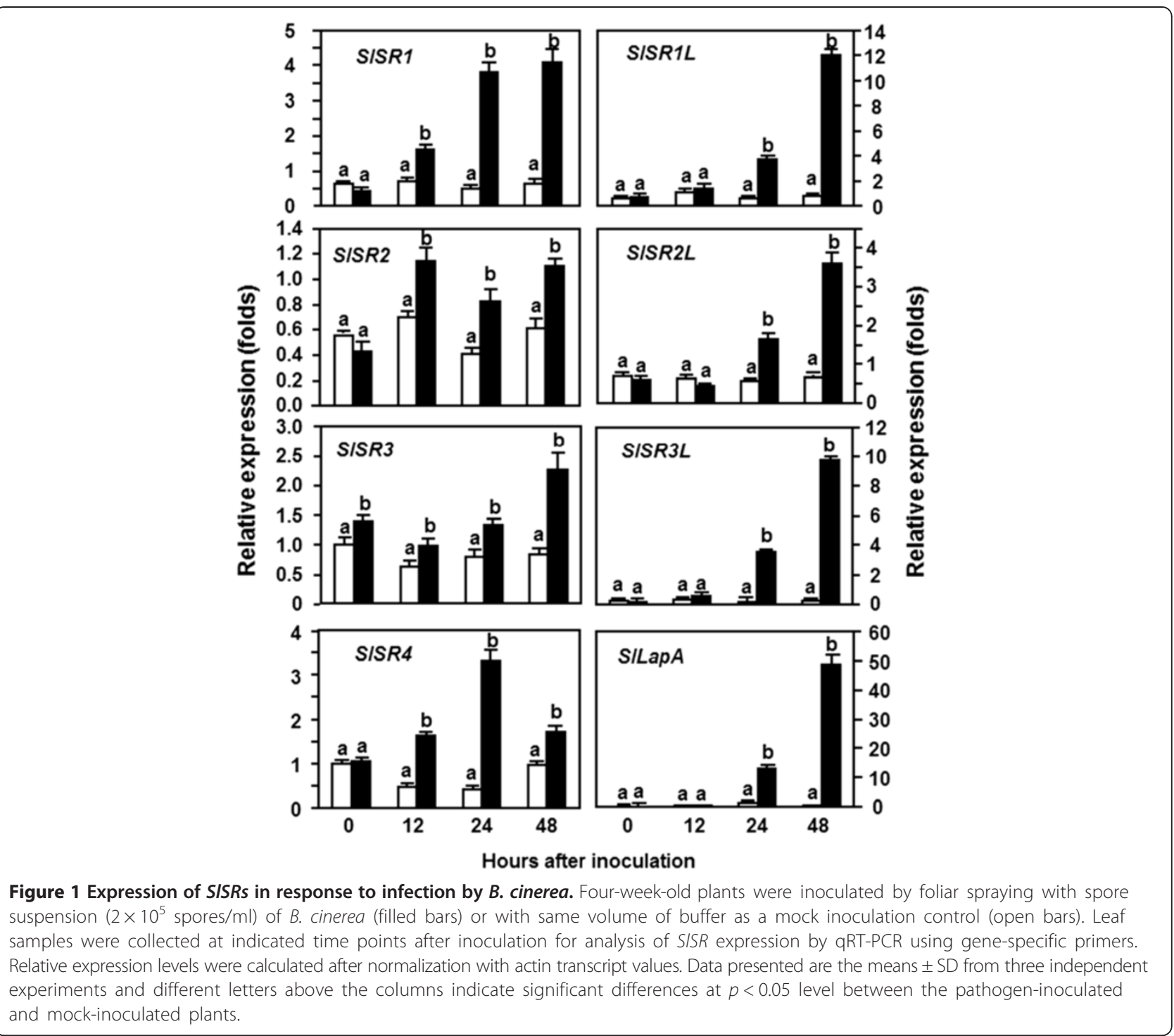

plants (Figure 1). These results indicate that the expression of SlSRs could be induced with distinct patterns by $B$. cinerea and that the expression of most SISR genes except for SISR2 and SISR3 was highly responsive to infection of $B$. cinerea.

We next analyzed the expression patterns of SISRs in response to a virulent strain of Pst DC3000. In these experiments, the expression pattern of SIPR-P2, a defense gene regulated by the SA-mediated signaling pathway [31], was examined to confirm the efficiency of the inoculation procedure. As shown in Figure 2, the expression level of SIPR-P2 in the Pst DC3000-inoculated plants increased at 6 hpi and showed 50 and $>300$ folds of increase at 12 and $24 \mathrm{hpi}$, over those in the mock-inoculated plants, confirming that the effectiveness of the inoculation procedure was satisfied for further analysis of the expression patterns of SlSRs in response to Pst DC3000. The expression levels of SISR1L, SISR2, SISR2L and SISR3 in the Pst DC3000-inoculated plants were similar to those in the mock-inoculated plants, indicating that their expression was not responsive to infection of Pst DC3000 (Figure 2). However, the expression levels of SISR1 and SISR3L were significantly induced by Pst DC3000 (Figure 2). A 3-fold increase of the expression of SISR1 in the Pst DC3000inoculated plants was observed at $24 \mathrm{hpi}$ but no significant increase in the expression level of SISR1 in the Pst DC3000-inoculated plants was observed within the first $12 \mathrm{hpi}$, as compared with those in the mock-inoculated plants (Figure 2). The expression level of SISR3L in the Pst DC3000-inoculated plants exhibited 2-fold and 4-fold increases at $12 \mathrm{hpi}$ and $24 \mathrm{hpi}$, respectively, relative to those in the mock-inoculated plants (Figure 2). Interestingly, the expression of SISR4 in the Pst DC3000-inoculated plants was suppressed by Pst DC3000 during the first $12 \mathrm{hpi}$ and 


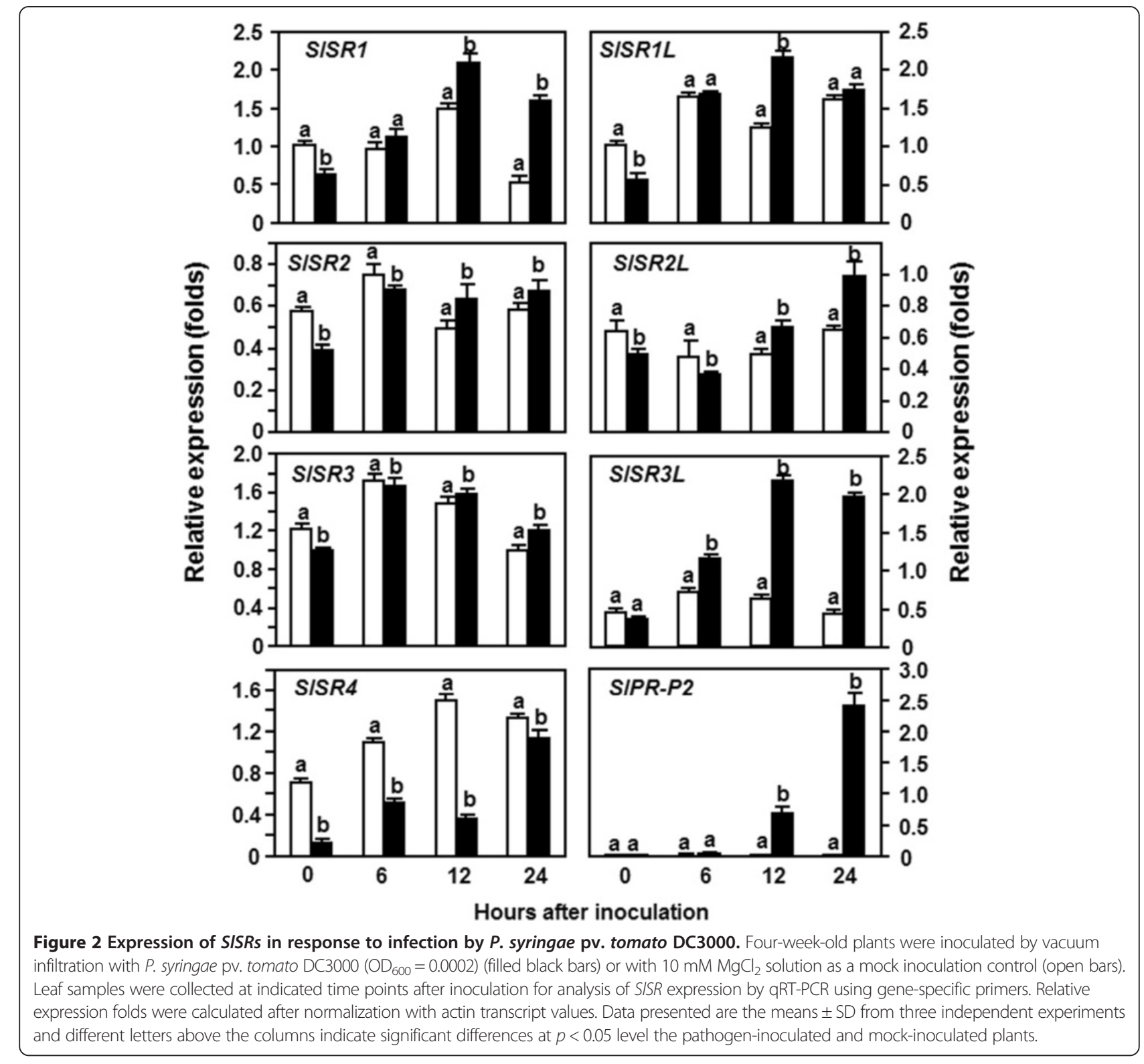

this suppression of SlSR4 expression was very quick as the expression level of SISR4 in the Pst DC3000-inoculated plants decreased by approximately 4 folds relative to that in the mock-inoculated plants (Figure 2). The expression level of SlSR4 in the Pst DC3000-inoculated plants restored to the level in the mock-inoculated plants (Figure 2). These results indicate that the expression of SISRs could be induced with distinct patterns by Pst DC3000 and that the expression of SISR1 and SISR3L was induced but the expression of SlSR4 was suppressed by Pst DC3000.

Silencing of SISR1 and SISR3L conferred an increased resistance to $B$. cinerea and Pst DC3000

To explore the possible functions of SlSRs in plant defense response, we used the TRV-based VIGS system
[32] to knockdown the expression levels of SISR genes in tomato plants and compared the phenotypes and severity of diseases caused by B. cinerea and Pst DC3000, respectively. In our VIGS study, we chose sequences that encode for the highly diverged regions in the SISR proteins to avoid interference with expression of other non-target SlSR genes when one target SISR gene was attempted to be silenced (Additional file 1). Silencing efficiency and specificity were assessed by qRT-PCR analyzing the transcript abundance of the target SISR gene in the silenced and non-silenced pTRV2-GUS-infiltrated control plants. As shown in Figure 3, the transcript levels of each SISR gene in the corresponding silenced plants were significantly reduced, leading to the silencing efficiency of 70-75\% in standard VIGS experiments, as compared with those 

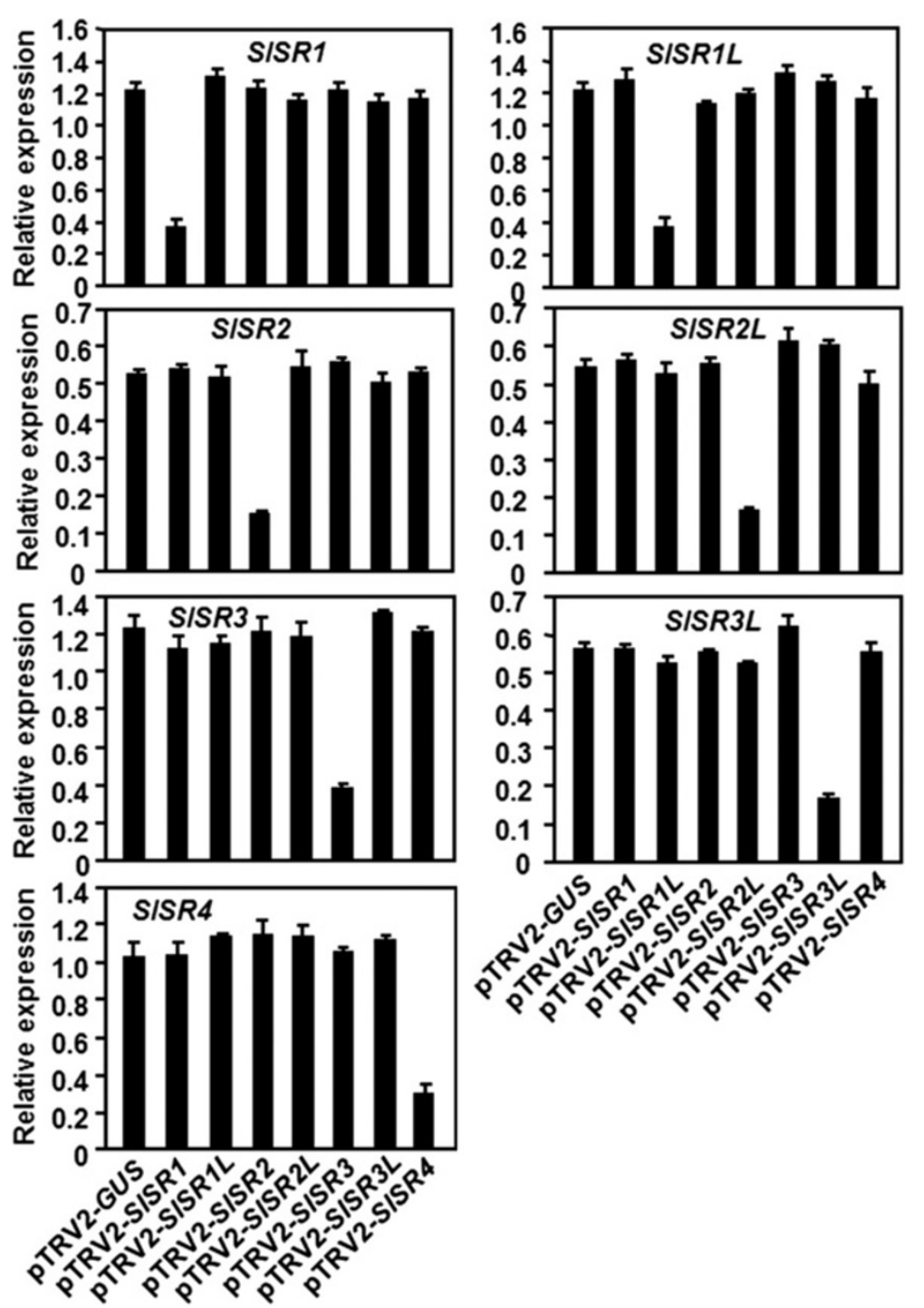

Figure 3 Silencing efficiency and specificity for target genes in silenced plants. Two-week-old tomato seedlings were infiltrated with agrobacteria carrying pTRV2-SISRs or pTRV2-GUS constructs and leaf samples were collected from pTRV2-SISRs- and pTRV2-GUS-infitlrated plants at 4 weeks after agroinfiltration. Expression levels of each SISR genes in targeted and nontargeted SISR genes-silenced and non-silenced plants were analyzed by qRT-PCR and data obtained were normalized with actin transcript values. Data presented are the means \pm SD from three independent experiments.

in the pTRV2-GUS-infiltrated plants. By contrast, the transcript levels of each SISR gene in those plants that were silenced for one of the other six SISR genes were comparable to the level in the pTRV2-GUS-infiltrated control plants (Figure 3), indicating that silencing only occurred for the targeted gene but not for the other nontargeted SISR genes. Thus, the silencing efficiency and specificity under our experiment conditions were satisfied for further study and all the subsequent experiments were performed only on those pTRV2-SISR-infiltrated plants with high levels of silencing efficiency $(>70 \%)$.

We first examined the possible roles of SlSRs in resistance against $B$. cinerea by challenging the pTRV2-SISRs- infiltrated plants with spore suspension of $B$. cinerea and comparing the disease severity and in planta fungal growth with those in pTRV-GUS-infiltrated non-silenced plants. In our detached leaf assays, $B$. cinerea-caused lesions on detached leaves from the pTRV2-SISR1L-, pTRV2-SISR2-, pTRV2-SISR2L-, pTRV2-SISR3- and pTRV2-SISR4-infiltrated plants were similar to the lesions on the detached leaves from pTRV2-GUS-infilrtratd plants (Figure 4A and B), suggesting that SISR1L, SISR2, SISR2L, SISR3 and SISR4 may not be involved in disease resistance against $B$. cinerea. However, $B$. cinerea-caused lesions on detached leaves from the pTRV2-SISR1- and pTRV2-SISR3L-infiltrated plans developed slowly and were still separated, as 

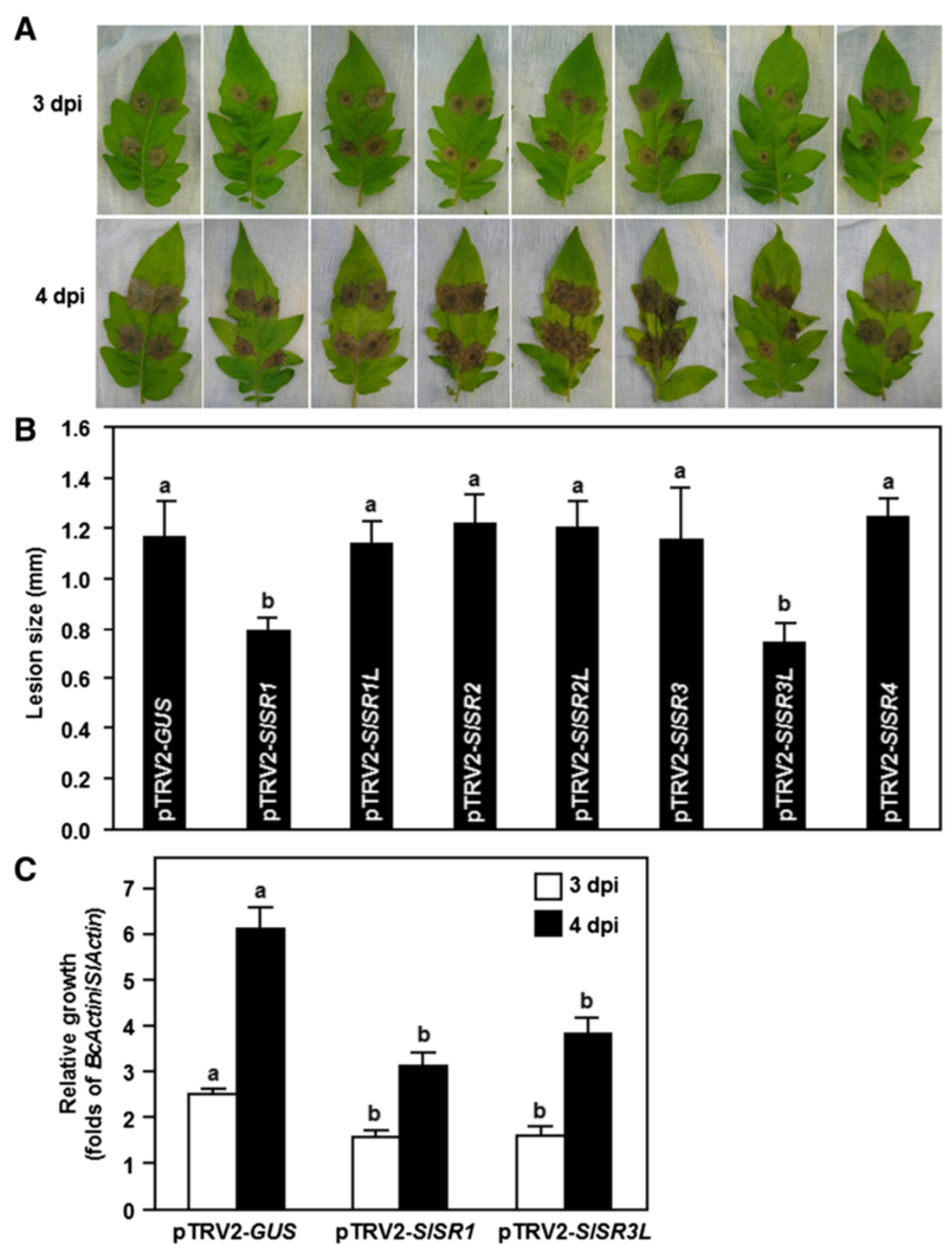

Figure 4 Silencing of either SISR1 or SISR3L resulted in enhanced disease resistance to $B$. cinerea. Two-week-old tomato seedlings were infiltrated with agrobacteria carrying pTRV2-SISRs or pTRV2-GUS constructs and leaves were detached from pTRV2-GUS- or pTRV2-SISR-infiltrated plants at 3 weeks after VIGS infiltration. Inoculation with B. cinerea was done by dropping $5 \mu$ lof spore suspension $\left(1 \times 10^{5}\right.$ spores $\left./ \mathrm{ml}\right)$. (A) Disease symptom on detached leaves at 3 days after inoculation. (B) Lesion size in leaves of the pTRV2-GUS- or pTRV2-SISR-infiltrated plants at 4 days after inoculation. At least 10 leaves from ten individual plants were used for each experiment. (C) Growth of B. cinerea in inoculated plants from whole plant inoculation experiments. Fungal growth in planta was assumed at 3 and 4 days after inoculation by qRT-PCR analyzing the transcript level of B. cinerea BcActinA gene using SIActin gene as an internal control. Relative fungal growth was shown as folds of transcript levels of BcActin compared to SIActin. Data presented are the means \pm SD from three independent experiments and different letters above the columns indicate significant differences at $p<0.05$ level.

compared with the large merged lesions on leaves from the pTRV2-GUS-infiltrated plants, at 4 days after inoculation (dpi) (Figure 4A). At 4 dpi, the lesion sizes on detached leaves from the pTRV2-SISR1- and pTRV2SISR3L-infiltrated plants were significantly reduced, leading to a reduction of approximately $35 \%$, as compared with that of the pTRV2-GUS-infiltrated plants (Figure 4B). We further analyzed and compared the in planta fungal growth in the pTRV2SISR1-, pTRV2-SISR3L- and pTRV2-GUS-infiltrated plants after inoculation by foliar spraying with spore suspension of $B$. cinerea in whole plant inoculation 
experiments. qRT-PCR analysis of the transcript levels of the B. cinerea actin gene BcActin, which was used as an indicative of the rate of fungal growth in planta, showed that the fungal growth in the pTRV2-SISR1- and pTRV2-SISR3L-infilrated plants were significantly suppressed, resulting in reductions of $35-51 \%$ at 3 and $4 \mathrm{dpi}$, as compared with those in the pTRV2-GUS-infiltrated plants (Figure 4C). These results indicate that the SISR1and SISR3L-silenced plants were more resistant to $B$. cinerea infection than the pTRV2-GUS-infiltrated plants. Taken together, these data demonstrate that silencing of SISR1 or SISR3L resulted in increased resistance against $B$. cinerea and thus both SISR1 and SISR3L may act as negative regulators of disease resistance against $B$. cinerea.

We next explored whether SlSRs have functions in resistance against Pst DC3000. Disease phenotypes and bacterial growth in planta were compared between the pTRV2-SISRs- and pTRV2-GUS-infiltrated plants after inoculation with a virulent strain of Pst DC3000. At 4 dpi, the pTRV2-GUS-infiltrated plants showed typical bacterial speck disease symptoms, including necrotic lesions surrounded by chlorosis (Figure 5A). The disease severity and bacterial growth in planta in inoculated pTRV2-SISR1L-, pTRV2-SISR2-, pTRV2-SISR2L-, pTRV2SISR3- and pTRV2-SISR4-infiltrated plants were comparable to those in the pTRV2-GUS-inflitrated plants (Figure 5A and B), suggesting that SISR1L, SISR2, SISR2L, SISR3 and SISR4 are not involved in disease resistance to Pst DC3000. By contrast, the pTRV2-SISR1and pTRV2-SISR3L-infiltrated plants showed very weak visible symptoms of disease caused by Pst DC3000, as compared with that in the pTRV2-GUS-infiltrated plants (Figure 5A). This reduced disease symptoms on leaves of the pTRV2-SISR1- and pTRV2-SISR3L-infiltrated plants were coincided with relatively low levels of bacterial growth in planta after inoculation with Pst DC3000. At 4 dpi, the bacterial populations in leaves of the pTRV2SISR1- and pTRV2-SISR3L-infiltrated plants were $2.8 \times$ $10^{5}$ and $2.2 \times 10^{5} \mathrm{CFU} / \mathrm{cm}^{2}$, leading to a reduction of 10 fold, as compared to that in leaves of the pTRV-GUSinfiltrated plants $\left(2.5 \times 10^{6} \mathrm{CFU} / \mathrm{cm}^{2}\right)$ (Figure $\left.5 \mathrm{~B}\right)$. These results demonstrate that silencing of either SISR1 or SlSR3L led to increased resistance against Pst DC3000 and thus both of SISR1 and SISR3L are also negative regulators of disease resistance to Pst DC3000.

\section{Silencing of SISR1 and SISR3L resulted in constitutive defense response}

The Arabidopsis sr1 mutant plants were previously found to display chlorosis and constitutive expression of

A

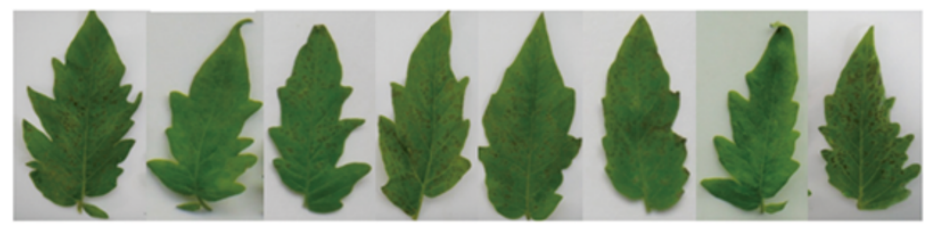

B

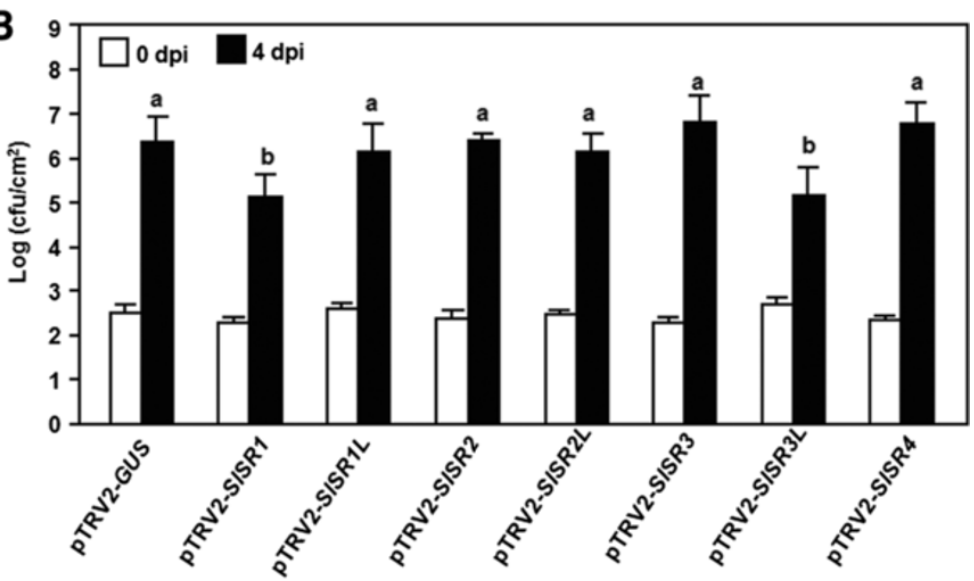

Figure 5 Silencing of either SISR1 or SISR3L led to enhanced resistance to $P$. syringae pv. tomato DC3000. Two-week-old tomato seedlings were infiltrated with agrobacteria carrying pTRV2-SISRs or pTRV2-GUS constructs and the pTRV2-GUS- and pTRV2-SISRs-infiltrated plants were inoculated by vacuum infiltration with infiltration with $P$. syringae pv. tomato $D C 3000\left(\mathrm{OD}_{600}=0.0002\right)$ at 3 weeks after VIGS infiltration. (A) Representative symptom of disease caused by P. syringae pv. tomato DC3000 at 4 days after inoculation. (B) Bacterial growth in inoculated leaves of pTRV2-GUS- and pTRV2-SISRs-infiltrated plants. Leaf samples were collected at 0 and 4 days after inoculation and bacterial growth was measured. Data presented are the means \pm SD from three independent experiments and different letters above the columns indicate significant differences at $p<0.05$ level. 
defense genes under lower temperature, indicating that loss of SR1 function in Arabidopsis led to constitutive defense response [20]. To examine whether silencing of SISR1 or SISR3L could also confer constitutive defense responses, we analyzed and compared the accumulation of $\mathrm{H}_{2} \mathrm{O}_{2}$ and expression of genes involved in different signaling pathways and defense response between the pTRV2-SISR1- and pTRV2-SISR3L-infiltrated plants and the pTRV2-GUS-infiltrated plants. Results from 3,3-diaminobenzidine (DAB) staining of in situ $\mathrm{H}_{2} \mathrm{O}_{2}$ accumulation showed that significant brown precipitates, representing the accumulation of $\mathrm{H}_{2} \mathrm{O}_{2}$, were easily and clearly observed in leaves of the pTRV2-SISR1- and pTRV2SISR3L-infiltrated plants without infection of pathogen, while no significant brown precipitate was seen in leaves of the pTRV2-GUS-infiltrated plants (Figure 6A). These results

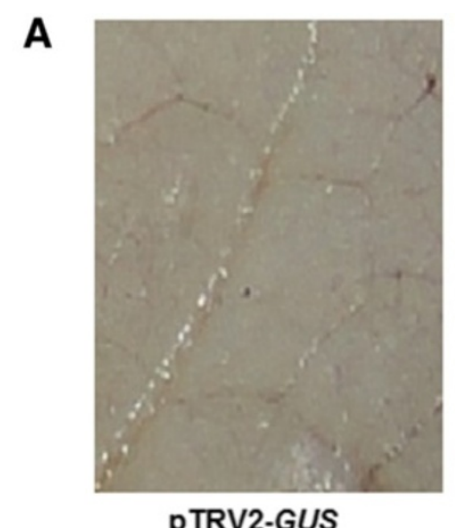

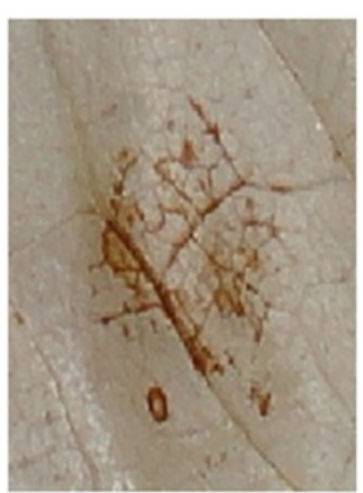

pTRV2-SISR1

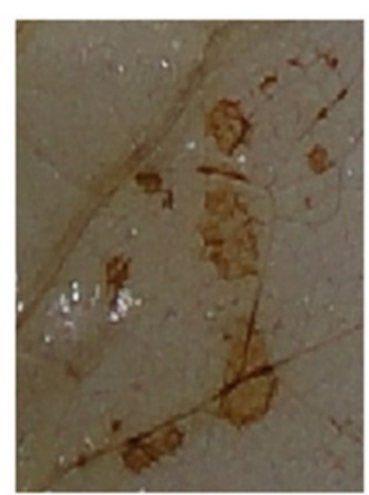

pTRV2-SISR3L
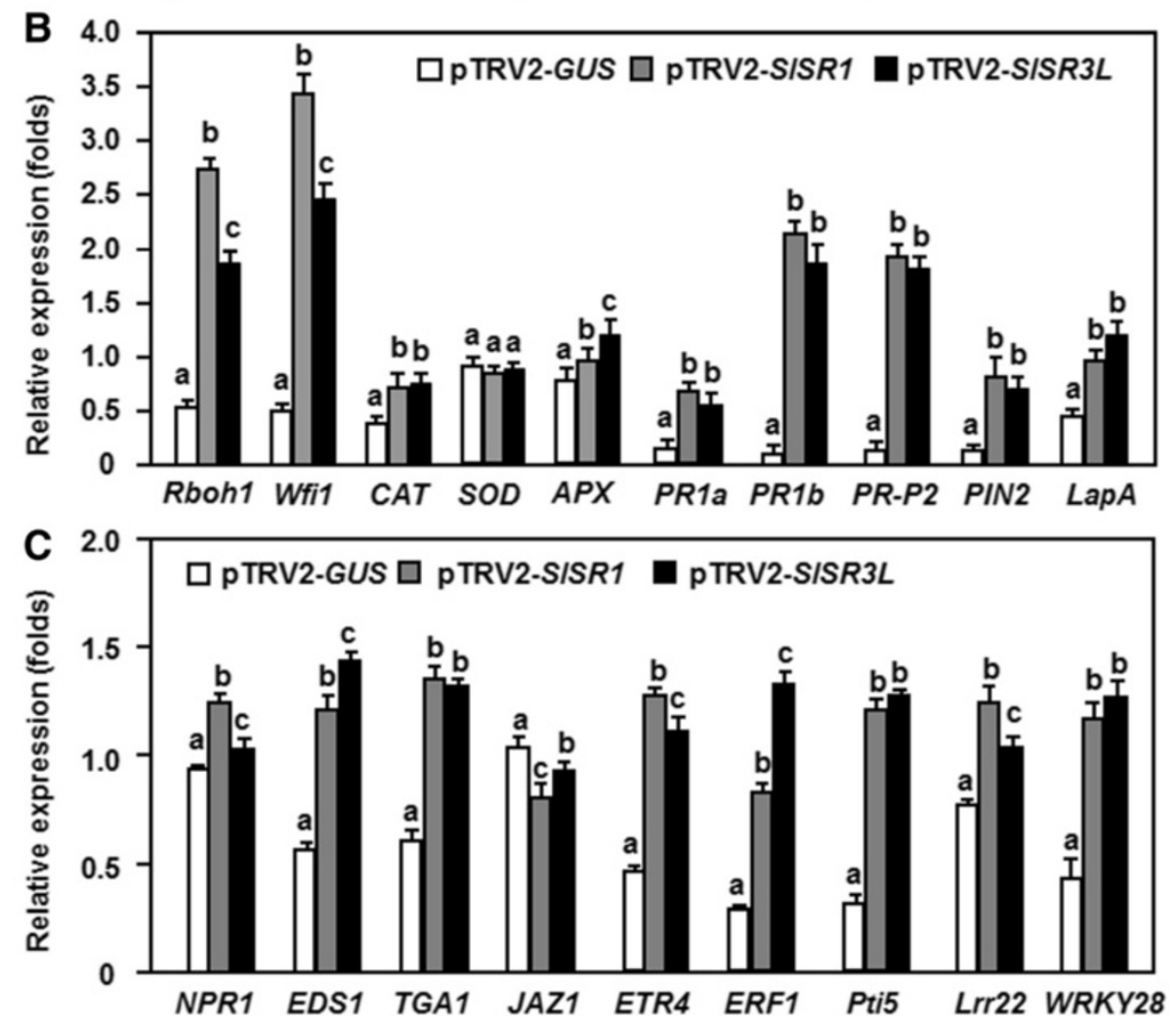

Figure 6 Silencing of either SISR1 or SISR3L activated constitutive defense response. Two-week-old seedlings were infiltrated with agrobacteria carrying pTRV2-SISR1, pTRV2-SISR3L or pTRV2-GUS constructs and leaf samples were collected at 2 weeks after VIGS infiltration for detection of $\mathrm{H}_{2} \mathrm{O}_{2}$ accumulation and analysis of expression of signaling- and defense-related genes. (A) Accumulation of $\mathrm{H}_{2} \mathrm{O}_{2}$ in leaves of the PTRV2-SISR1- and PTRV2-SISR3L-infiltrated plants. (B) Expression of defense-related genes and ROS production-related genes in the PTRV2-SISR1- and pTRV2-SISR3L-infiltrated plants. (C) Expression of genes involved in different defense signaling pathways in the pTRV2-SISR1- and pTRV2-SISR3L-infiltrated plants. Data presented are the means \pm SD from three independent experiments and different letters above the columns indicate significant differences at $p<0.05$ level. 
indicate that silencing of either SISR1 or SISR3L resulted in constitutive accumulation of $\mathrm{H}_{2} \mathrm{O}_{2}$ in tomato plants. To explore the possible mechanism for the constitutive accumulation of $\mathrm{H}_{2} \mathrm{O}_{2}$ in the SISR1- and SISR3L-silencedd plants, we analyzed and compared the expression of genes encoding for NADPH oxidases, which are plasma membranelocalized ROS generating enzymes [33], and for catalases (CAT), superoxide dismutases (SOD) and ascorbate peroxidases (APX), which are involved in scavenging of ROS, in the pTRV2-SISR1- and pTRV2-SISR3L-infiltrated plants. As shown in Figure 6B, the expression levels of Rboh1 and Wfil, two genes for NADPH oxidases, in the pTRV2-SISR1- and pTRV2-SISR3L-infiltrated plants were significantly elevated, giving increases of $5 \sim 7$-fold for Rboh1 and 4 5-fold for Wfil, as compared with those in the pTRV2-GUS-infiltrated plants. Similarly, the expression levels of $C A T$ and $A P X$ in the pTRV2-SISR1- and pTRV2-SISR3L-infiltrated plants were also increased as compared with those in the pTRV2-GUS-infiltrated plants (Figure 6B). By contrast, no significant difference was observed in the expression level of $S O D$ between the pTRV2-SISR1- and pTRV2-SISR3L-infiltrated plants and the pTRV2-GUS-infiltrated plants (Figure 6B). These results indicate that the constitutive accumulation of $\mathrm{H}_{2} \mathrm{O}_{2}$ in the SISR1- and SISR3L-silenced plants might be attributed to the increased ROS generating ability resulted from the high level of expression of the NADPH oxidases.

We next examined whether silencing of SISR1 or SISR3L led to constitutive expression of defense genes. The expression levels of five representative defense genes that are regulated through different defense signaling pathways and three marker genes for pathogen-associated molecular patterns-triggered immunity (PTI) were analyzed and compared between the pTRV2-SISR1- and pTRV2-SISR3Linfiltrated plants and the pTRV2-GUS-infiltrated plants. As shown in Figure 6B, the expression levels of PR1a, PR1b and $P R-P 2$, which are thought to be regulated by the SAmediated signaling pathway [31], and of PIN2 and LapA, which are considered to be regulated by the JA/ET signaling pathway [31], were increased significantly in the pTRV2SISR1- and pTRV2-SISR3L-infiltrated plants, leading to 8 $\sim 25$ folds of increases for the $P R 1 a, P R 1 b$ and $P R-P 2$ genes and $1 \sim 11$ folds of increases for the PIN2 and LapA genes, as compared with those in the pTRV2-GUS-infiltrated plants. Similarly, the expression levels of Pti5, Lrr22 and WRKY28, three PTI marker genes in tomato $[34,35]$, in the pTRV2-SISR1- and pTRV2-SISR3L-infiltrated plants were significantly higher than those in the pTRV2-GUS-infiltrated plants, resulting in increases of $0.5 \sim 3.0$ folds in the expression levels (Figure $6 \mathrm{C}$ ). These data demonstrate that silencing of either SISR1 or SISR3L activates constitutively the expression of the defense and PTI genes, leading to constitutive defense response in the SISR1- and SISR3L-silenced plants.
To determine whether silencing of SISR 1 and SISR $3 L$ activates defense signaling pathways, we further analyzed and compared the expression levels of the key genes encoding important components involved in the SA- and JA/ET-mediated signaling pathways between the pTRV2SISR1- and pTRV2-SISR3L-infiltrated plants and the pTRV2-GUS-infiltrated plants. The expression levels of NPR1, EDS1 and TGA1, known to be critical components in the SA-mediated signaling pathway [36], in the pTRV2SISR1- and pTRV2-SISR3L-infiltrated plants were significantly increased, especially for the expression levels of the EDS1 and TGA1 genes, showing 1-fold increase relative to those in the pTRV2-GUS-infiltrated plants (Figure 6C). Similarly, the expression levels of ETR4 and ERF1, known to be associated with ET signaling pathway $[37,38]$, in the pTRV2-SISR1- and pTRV2-SISR3L-infiltrated plants showed 1 2-fold increase over those in the pTRV2GUS-infiltrated plants (Figure 6C). By contrast, the expression level of $J A Z 1$, known to be associated with JA signaling pathway [39], in the pTRV2-SISR1- and pTRVSISR3L-infiltrated plants was reduced as compared with those in the pTRV2-GUS-infiltrated plants (Figure 6C). These results suggest that silencing of either SISR1 or SISR3L can activate both the SA- and ET-mediated signaling pathways but suppress the JA-mediated signaling pathway in the SISR1- and SISR3L-silenced plants.

\section{Expression patterns of SISRs in response to drought stress and $A B A$}

To explore the possible involvement of SlSRs in drought stress response, we first examined whether the expression of SlSRs could be induced by drought stress and abscisci acid (ABA) treatment. Two different methods, the detached leaf and the whole plant assays, were adapted to analyze the expression of SlSRs in response to drought stress. In these experiments, a previously reported drought stress-responsive gene, SGN-213276 [40], was included to confirm the efficiency of the drought stress treatment. The expression level of SGN-213276 was markedly increased after drought stress treatment, leading to increases of $>100$-fold at $5 \mathrm{hr}$ after treatment in the detached leaf assays (Figure 7A) and at 7 days after treatment in the whole plant assays (Figure 7D), indicating that the experiments for drought stress assays were satisfied for further analyzing the expression of the SISR genes. In the detached leaf assays, as compared with the expression levels of the corresponding genes in the water-saturated detached leaves, no significant change in the expression levels of SISR1, SISR2, SISR3 and SISR4 was observed over a period of $5 \mathrm{hr}$ after detachment (Figure 7A), whereas a maximal increase of $4 \sim 8$-fold in the expression levels of SISR2L and SISR3L was observed during 3-5 hr after treatment (Figure 7A). By contrast, the expression kinetic of SISR1L under drought stress condition was similar to 

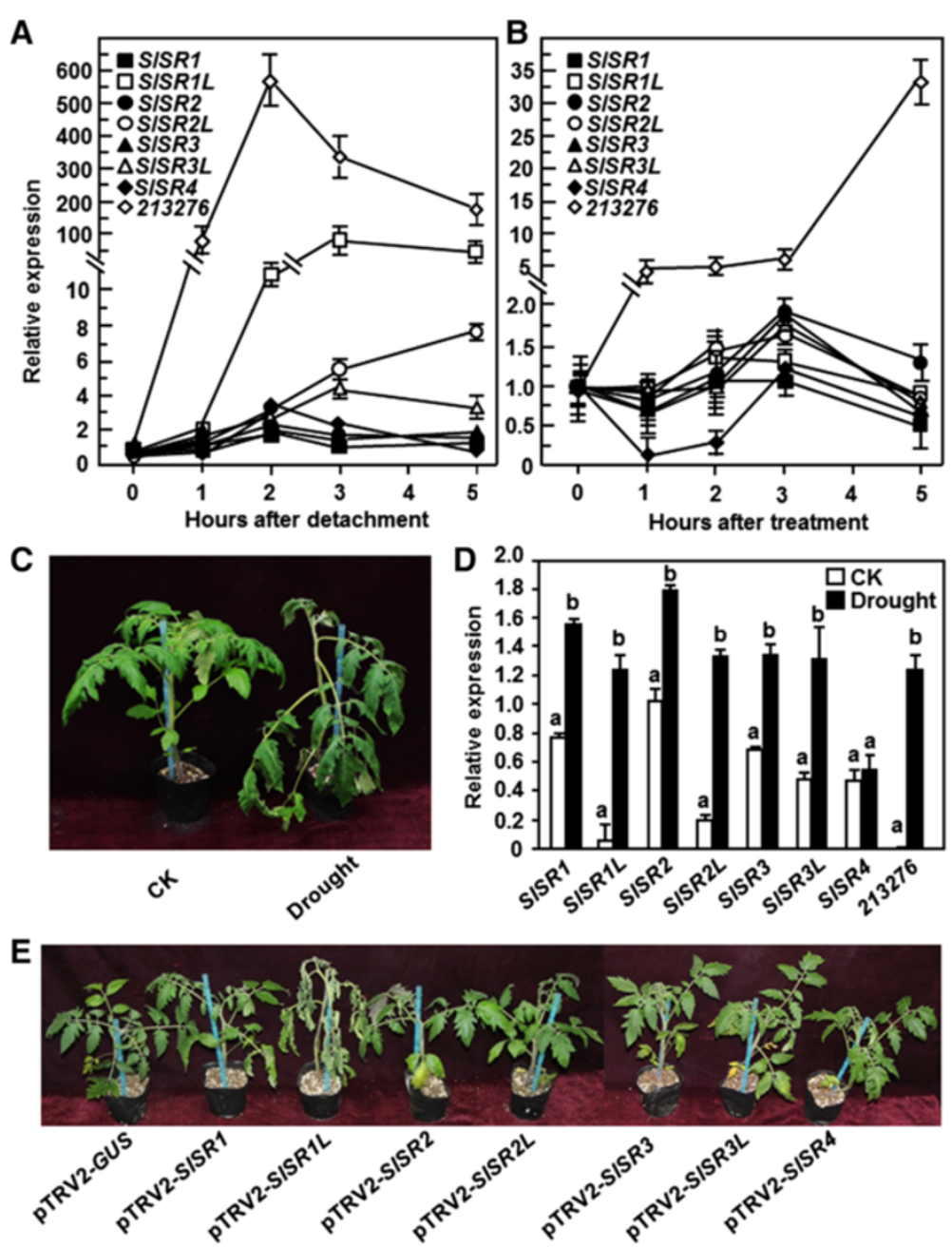

Figure 7 Expression patterns of SISRs in response to drought stress and ABA treatment and phenotypes of the SISRs-silenced plants under drought condition. (A) Expression of SISRs in detached leaves under drought stress. Fully expanded leaves were detached from four-week-old plants and subjected to drought stress treatment by placing on lab blench or water-saturated filter papers in Petri dishes as a control and samples were collected at different time points as indicated. (B) Expression of SISRs in detached leaves after ABA treatment. Four-week-old plants were treated by foliar spraying with ABA solution $(100 \mu \mathrm{M})$ or water as control and leaf samples were collected at different time points as indicated. Relative expression levels of the SISR genes in the treated plants were shown as folds of the expression levels in the control plants after normalization with actin transcript values. (C) and (D) Expression of SISRs in leaves of plants under drought stress. Four-week-old plants were treated for drought stress by stopping watering for a period or watered normally as controls and leaf samples were collected at 7 days after treatment when wilting symptom appeared (C). Total RNA was extracted and used for qRT-PCR analysis of expression of SISRs (D). Relative expression levels of the SISR genes in the treated and control plants were shown as folds of the actin transcript values. (E) Phenotypes of the SISRs-silenced plants under drought condition. Two-week-old seedlings were infiltrated with agrobacteria carrying pTRV2-SISRS or pTRV2-GUS constructs and 3 weeks later the pTRV2-GUS- and pTRV2-SISRs-infiltrated plants were subjected to drought stress by stopping watering for 10 days. Data presented in (A), (B) and (D) are the means \pm SD from three independent experiments and different letters above the columns in $(\mathbf{D})$ indicate significant difference at $p<0.05$ level.

that of the SGN-213276 gene (Figure 7A). The expression level of SISR1L in the drought stress treated leaves started to increase at $2 \mathrm{hr}$ after detachment and exhibited approximately an increase of $50 \sim 90$ folds as compared with that in the water-saturated leaves (Figure 7A). In the whole plant assays, the drought stressed plants exhibited clear wilting symptom at 7 days after withholding water whereas the normally watered plants did not show any wilting symptom (Figure 7C). qRT-PCR analyses revealed that the expression of SISRs except SISR4 was induced by drought stress. The expression levels of SISR1, SISR2, SISR3 and SISR3L in the drought stressed plants showed a slight increase, leading to an increase of approximately 1 fold or less over those in the normally watered plants (Figure 7D). However, the expression levels of SISR1L and SISR2L in the drought stressed plants increased significantly as compared with those in the normally watered plants, resulting in increases of 20 folds and 7 folds, respectively 
(Figure 7D). Taken together, these results suggest that some SISRs especially SISR1L are responsive to drought stress. We also analyzed whether the expression of SISRs could be induced by exogenous application of ABA, a wellknown hormone involved in drought stress response [41]. In these experiments, the expression level of the drought stress-responsive gene SGN-213276 in the ABA-treated plants was markedly increased, leading to increases of 5 34-fold over those in the control plants (Figure 7B); however, the expression levels of SISRs in the ABA-treated plants showed changes with less 2 folds as compared with those in the control plants (Figure 7B), indicating that exogenous ABA did not affect the expression of SISRs.
Silencing of SISR $1 L$ reduced drought tolerance in tomato VIGS assays were performed to explore the involvement of individual SlSRs in drought stress response. For this purpose, we stopped watering for 7-10 days to compare the phenotype between the pTRV2-SISRs- and pTRV2GUS-inflitrated plants. In repeated experiments, silencing of SlSR1, SlSR2, SlSR2L, SlSR3, SlSR3L or SlSR4 did not affect drought stress response over an experimental period of 10 days (Figure 7E), indicating that these SlSR genes may not be involved in drought stress response. However, the pTRV-SISR1L-infiltrated plants showed significant wilting symptom at 7 days after drought stress treatment as compared with that of the pTRV2-GUSinfiltrated plants (Figure 7E), implying a role for SlSR1L in
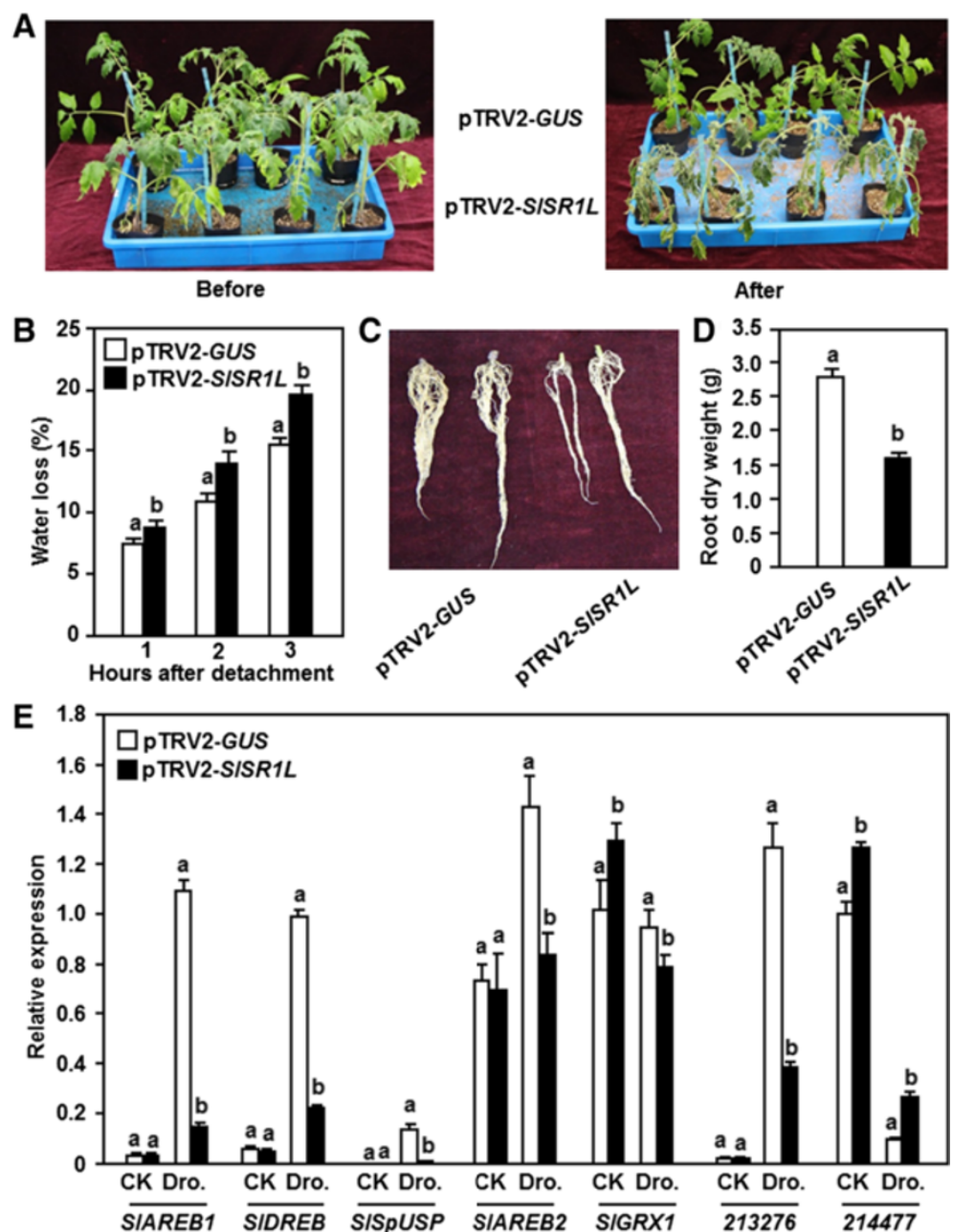

Figure 8 Silencing of SISRIL led to reduced drought stress tolerance. Two-week-old tomato seedlings were infiltrated with agrobacteria carrying PTRV2-SISR1L or PTRV2-GUS constructs and 3 weeks later the PTRV2-GUS- and PTRV2-SISR1L-infiltrated plants were subjected to drought stress by stopping watering. (A) Phenotype of the pTRV2-GUS- and PTRV2-SISR1L-infiltrated plants before and after treatment of drought stress. (B) Rates of water loss in detached leaves of the pTRV2-GUS- and pTRV2-SISR1L-infiltrated plants. (C) Root system of the pTRV2-GUS- and pTRV2-SISR1L-infiltrated plants. The intact root systems are shown from one representative pTRV2-GUS- or pTRV2-SISR1L-infiltrated plant. (D) Dry weights of roots from the pTRV2-GUS- and PTRV2-SISR1L-infiltrated plants. (E) Expression of some drought-responsive genes in the pTRV2-GUS- and pTRV2-SISR1L-infiltrated plants before and after treatment of drought stress. Data presented in (B), (C) and (E) are the means \pm SD from three independent experiments and different letters above the columns indicate significant differences at $p<0.05$ level. 
drought stress tolerance. The pTRV2-SISR1L-infiltrated plants grew well as the pTRV2-GUS-infiltrated plants before withholding water, but they were easier to appear wilting symptom after stopping watering and their leaves became curly and the plants wilted at 7 days after withholding water (Figure 8A). This result indicates that silencing of SISR1L attenuated the drought stress tolerance in tomato. To explore the possible mechanism for the reduced drought stress tolerance in the $S I S R 1 L$-silenced plants, we first analyzed and compared the physiological and morphological changes between the pTRV2-SISR1L- and pTRV2GUS-infiltrated plants before and after drought stress treatment. The rate of water loss in leaves from the pTRV2-SISR1L-infiltrated plants was higher than those in leaves from the pTRV2-GUS-infiltrated plants during the first $3 \mathrm{hr}$ after detachment (Figure 8B), indicating that silencing of SISR1L accelerated water loss in leaves. The pTRV2-SISR1L-infiltrated plants had smaller root system as compared to the pTRV2GUS-infiltrated plants (Figure 8C). Similarly, dry weights of the roots from the pTRV2-SISR1Linfiltrated plants were significantly lower than that of the roots from the pTRV2-GUS-infiltrated plants, resulting in a reduction of approximately 40\% (Figure 8D). Furthermore, we also analyzed and compared the expression of some previously reported drought stress-responsive genes SIAREB1 [42], SlAREB2 [42], SIDREB [43], SlSpUSP [44], SlGRX1 [45], SGN213276 [40] and SGN-214777 [40] in the pTRV2SISR1L- and pTRV2-GUS-infiltrated plants before and after drought stress treatment. Before drought stress treatment, the expression levels of SIAREB1, SIAREB2, SIDREB, SISpUSP and SGN-213276 in the pTRV2SISR1L-infiltrated plants were comparable to those in the pTRV2-GUS-infiltrated plants, whereas the expression levels of SlGRX1 and SGN-214777 the pTRV2SISR1L-infiltrated plants showed a slight increase as compared with those in the pTRV2-GUS-infiltrated plants (Figure 8E). At 10 days after drought stress treatment, the expression levels of SIAREB1, SIDREB, SISpUSP, SIAREB2 and SGN-213276 in the pTRV2-GUS-infiltrated plants increased significantly as compared with those in the normally watered control plants (Figure 8E). After drought stress treatment, however, the expression levels of SIAREB1, SIDREB, SISPUSP, SIAREB2 and SGN-213276 in the pTRV2-SISR1L-infiltrated plants decreased markedly as compared with those in the pTRV2-GUS-infiltrated plants (Figure 8E). The expression level of SGN-214777 decreased in the PTRV2-GUS-infiltrated plants as compared with that in the normally watered plants but increased significantly in the pTRV2-SISR1L-infiltrated plants as compared with that in the pTRV2-GUS-infiltrated plants after drought stress treatment (Figure 8E).

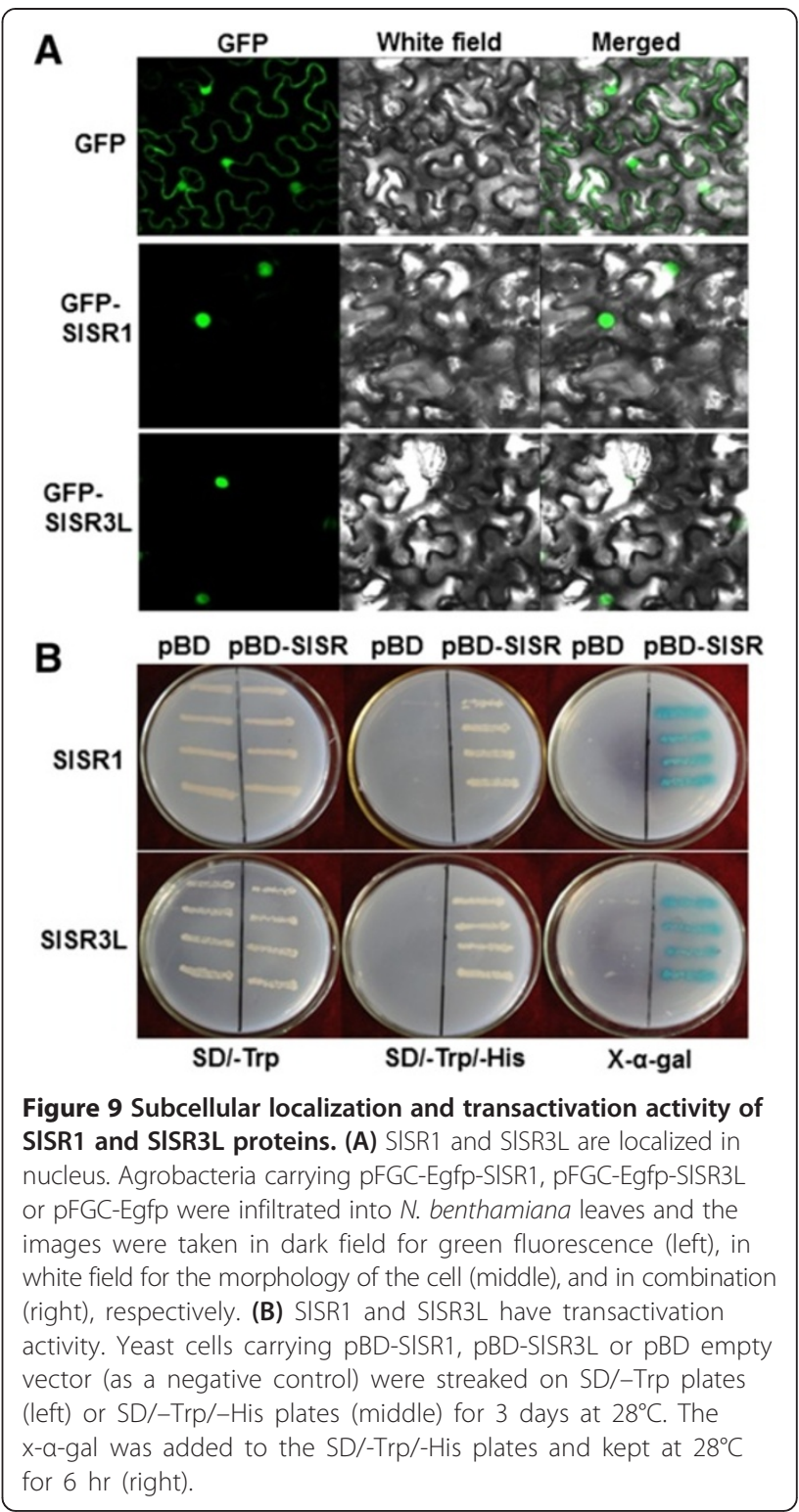

SISR 1 and SISR3L are localized in nucleus and have transactivation activity

Because of the importance of SISR1 and SISR3L in defense response against $B$. cinerea and Pst DC3000, we investigated the biochemical characteristics of these two SISR proteins. Firstly, we examined the subcellular localization of SISR1 and SISR3L using a transient expression approach. We transiently expressed SISR1 and SISR3L in leaves of 4-week-old $N$. benthamiana plants by infiltration with agrobacteria carrying pFGC-Egfp-SISR1, pFGC-EgfpSISR3L or pFGC-Egfp constructs and GFP was observed at 2 days after agroinfiltration. As shown in Figure 9A, the SISR1-GFP and SISR3L-GFP fusions accumulated exclusively in the nucleus of $N$. benthamiana cells, whereas the GFP protein alone accumulated in both the cytoplasm and 
the nucleus, demonstrating that both SISR1 and SISR3L proteins are localized in the nucleus of cells. Furthermore, we also examined whether the SISR1 and SISR3L proteins had transactivation activity using a yeast assay system. As shown in Figure 9B, all yeast transformants grew well on $\mathrm{SD} /-$ Trp medium. However, only yeast transformants containing pBD-SISR1 or pBD-SISR3L were able to grow on the $\mathrm{SD} /-\mathrm{Trp} /-$ His medium and produced a blue pigment after the addition of $x$ - $\alpha$-gal, showing a $\beta$-galactosidase activity, whereas transformants containing the pBD empty vector did not. These results indicate that both SISR1 and SISR3L have transactivation activity in yeasts. Taken together, our experimental data demonstrate that both SISR1 and SISR3L are nucleus-localized transcriptional activators.

\section{Discussion}

Previous studies have identified a total of seven SISRs genes in tomato and found that expression of SlSRs was regulated in tomato fruit by developmental cues and by biotic and abiotic environmental stress signals [29,30]; however, the biological functions of SlSRs in tomato response to biotic and abiotic stress remain unclear. In the present study, data from our VIGS-based functional analyses demonstrate that both SISR1 and SISR3L act as negative regulators of defense response against $B$. cinerea and Pst DC3000 while SISR1L functions as a positive regulator of drought stress tolerance in tomato. These findings not only demonstrate that members of the small SISR family play important roles in regulation of defense responses to biotic and abiotic stresses but also extend our understanding on the biological function of SlSRs in regulating stress response.

In plants, members of the SR family have been shown to be induced by infections from different pathogens. For example, the expression of AtSR1 in Arabidopsis was induced by Pst DC3000 and G. cichoracearum [21]. In this study, we observed that the expression of SlSRs could be induced with different patterns by Pst DC3000 and B. cinerea (Figures 1 and 2). In response to infection of $B$. cinerea, all the SISRs showed upregulated expression patterns in leaf tissues, especially for SISR1, SISR $1 L$, SISR3L and SISR4, whose expression levels were significantly upregulated (Figure 1). The B. cinerea-induced expression patterns of SISRs in leaf tissues are somewhat different from the previous observation that the expressions of SISR1, SISR1L, SISR2L and SISR3 in wounded tomato fruit were suppressed by $B$. cinerea [30], probably due to the tissue-specific expression feature of the SISRs [29]. In contrast to the B. cinerea-induced expression patterns of SlSRs, expression of SISR1, SlSR1L, SISR3 and SISR3L in leaf tissues was upregulated in response to infection of Pst DC3000 (Figure 2). The Pst DC3000induced expression of SISR1 is similar to that of the Arabidopsis AtSR1, closely related to SISR1 [29], whose expression was induced by Pst DC3000 [21]. However, infection of Pst DC3000 did not affect the expression of SlSR2 and SlSR3 but suppressed the expression of SlSR4 in leaf tissues (Figure 2), which is contrast to the observation that the expression of SISR4 in fruit tissues was induced by B. cinerea [30]. Therefore, it seems reasonable that the expression of SISRs is precisely controlled by complex mechanisms in response to infection from different pathogens. On the other hand, the B. cinerea- and Pst DC3000-induced expressions of SISRs, especially for SISR1 and SISR3L, were much evident after $24 \mathrm{hr}$ of inoculation (Figures 1 and 2), which is similar to the observation that the expression of AtSR1 in Arabidopsis leaves was only significantly induced by Pst DC3000 after $24 \mathrm{hr}$ of inoculation [21]. It was also found that the expression of most SISRs in tomato fruits was markedly induced by exogenous SA and MeJA only after $8 \mathrm{hr}$ of treatment [30]. These observations suggest that most of the SlSRs should belong to late pathogen-responsive genes [30]. The B. cinerea- and Pst DC3000-induced expression of SISR1 and SISR3L in leaves (Figures 1 and 2), along with their SA- and MeJA-induced expression patterns in fruits [30], suggest that SISR1 and SISR3L may play important roles in defense response to infection of $B$. cinerea and Pst DC3000.

In our VIGS-based functional analyses of SlSRs in defense response against different pathogens, we found that silencing of either SISR1 or SISR3L resulted in increased disease resistance against B. cinerea and Pst DC3000, as the SISR1and SISR3L-silenced plants exhibited less severity of the diseases and supported less in planta growth of the pathogens than the non-silenced plants (Figures 4 and 5), indicating that loss-of-function of either SISR1 or SISR3L confers a broad-spectrum disease resistance against different pathogens with distinct infection styles. SISR1 is phylogenetically closely related to the Arabidopsis AtSR1 [29], whose loss-of-function mutant plants showed increased disease resistance against three different pathogens including Pst DC3000, B. cinerea and G. cichoracearum [19-21] and gain-of-function mutant plants exhibited compromised systemic acquired resistance and basal immunity $[21,24]$. Similar results were also observed in the mutant plants of the $O S C B T$ gene, a rice SR family member closely related to AtSR1 and SISR1, which exhibited significant enhanced resistance to blast fungal pathogen M. grisea and leaf blight bacterial pathogen X. oryzae pv. oryzae [17]. Thus, it is likely that loss-of-function of some of the SR family members can confer broadspectrum disease resistance in plants. This may have extensive significance that members of the SR family can be used for generating transgenic varieties with broad-spectrum resistance in economically important crops through RNA interference-mediated suppression of expression of target SR genes. Notably, in addition to SR1, the function of 
other SR family members in disease resistance has not been elucidated so far. In this study, we demonstrated that SISR3L, like SISR1 in tomato (Figures 4 and 5) and AtSR1 in Arabidopsis [19-21], acts as a negative regulator of disease resistance against different pathogens in tomato. This finding characterized one member with important function in disease resistance from the relatively small SR family. It will be interesting to examine whether the Arabidopsis homologs of SISR3L, AtSR3 and AtSR6 [29], play roles in regulating disease resistance. Although expression of SISR1L, SISR2, SISR2L and SISR3 was induced by B. cinerea and/or Pst DC3000 (Figures 1 and 2), silencing of each of these SISR genes had no effect on disease resistance against these two pathogens (Figures 4 and 5). The expression of SISR4 was induced by B. cinerea but suppressed by Pst DC3000 (Figures 1 and 2); however, silencing of SISR4 did not lead to any alteration in disease resistance to B. cinerea and Pst DC3000 (Figures 4 and 5). These results indicate that the SISR genes are highly responsive to pathogen infection but the responsiveness of these SISR genes may be a side effect caused by pathogen infection but not a true reflection for their function in disease resistance.

The observed increased disease resistance against $B$. cinerea and Pst DC3000 in the SISR1- and SISR3L-silenced plants may be attributed to an improved basic immunity resulted from loss-of-function of SISR1 and SISR3L. This hypothesis is supported by several lines of evidence obtained from some biochemical and molecular analyses toward the SISR1- and SISR3L-silenced plants under pathogen-free conditions. Firstly, the SISR1- and SISR3Lsilenced plants constitutively accumulated high level of $\mathrm{H}_{2} \mathrm{O}_{2}$ in leaves, as revealed by in situ DAB staining (Figure 6A). Similar phenomenon was also observed in the Arabidopsis $s r 1$ mutant plants that accumulated high level of $\mathrm{H}_{2} \mathrm{O}_{2}$ in leaves without pathogen infection [19,20]. It was previously found that suppression of SlWfil expression resulted in significant decrease of $\mathrm{H}_{2} \mathrm{O}_{2}$ accumulation in antisense tomato plants [46]. The expression of SlWfil and SlRboh1, encoding plasma membrane-localized NADPH oxidases that are involved in generating ROS [33], was significantly upregulated in the SISR1- and SISR3L-silenced plants (Figure 6B), indicating an accelerated generation of ROS in these plants. By contrast, the ROS scavenging capacity in the SISR1- and SISR3L-silenced plants might not be affected significantly as the expression of the $S O D$ gene was not changed (Figure 6B). The upregulated expression of the CAT and APX genes in the SISR1- and SISR3Lsilenced plants might be a response to the high level of $\mathrm{H}_{2} \mathrm{O}_{2}$ accumulated in these plants as $\mathrm{H}_{2} \mathrm{O}_{2}$ was found to be capable of mediating the expression of CAT genes under stress conditions $[47,48]$. An accelerated generation with an unchanged scavenging capacity in the SISR1- and SISR3L-silenced plants broke the ROS generating and scavenging balance and favored to accumulate high level of $\mathrm{H}_{2} \mathrm{O}_{2}$, which in turn acts as signaling molecules to activate defense response against pathogens. Secondly, the SISR1- and SISR3L-silenced plants constitutively expressed defense genes and PTI marker genes. Similar to the observations that the Arabidopsis $s r 1$ and rice oscbt mutant plants constitutively expressed a diverse set of defense genes $[17,19,20]$, constitutive high levels of expression of defense genes including SIPR1a, SIPR1b, SIPR-P2, SIPIN2 and SlLapA, which are regulated by different signaling pathways, were observed in the SISR1- and SISR3Lsilenced plants (Figure 6B). In addition, the expression of the PTI marker genes including Pti5, Lrr22 and WRKY28 $[34,35]$ was also significantly upregulated in the SISR1and SISR3L-silenced plants (Figure 6C). These data indicate that both SISR1 and SISR3L negatively regulate PTI response. In this regarding, silencing of either SISR1 or SISR3L would relieve their suppression on PTI response and thus lead to increased resistance against multiple pathogens including B. cinerea and Pst DC3000 (Figures 4 and 5). This is supported by general knowledge that PTI but not effector-triggered immunity (ETI) plays important roles in regulating immunity against necrotrophic fungal pathogens like B. cinerea [49] while both the PTI and ETI are require for immunity to biotrphic/ hemibiotrophic pathogens such as Pst DC3000 [50]. Thirdly, the SISR1- and SISR3L-silenced plants constitutively activated the SA- and ET-mediated defense signaling pathways. It was previously found that the Arabidopsis $s r 1$ mutant plants contained high level of SA and had upregulated expression of defense and signaling genes $[19,20]$ and that AtSR1 can bind to the CGCG box in the promoters of EDS1, NDR1 and EIN3 [20,21], indicating the involvements of AtSR1 in the SA- and ET-mediated signaling pathways. Our qRT-PCR analyses of expression of some defense signaling pathway-associated genes also demonstrated that both SISR1 and SISR3L have functions that negatively regulate the SA- and ETmediated signaling pathways in tomato, as revealed by the upregulated expression of the SINPR1, SIEDS1, SITGA1, SlETR4 and SlERF1 genes in the SlSR1- and SlSR3L-silenced plants (Figure 6C). This is partially supported by the facts that the expression of SISR 1 and SISR $3 L$ in tomato fruits was induced rapidly by exogenously applied SA and ET [29,30]. In particular, it was shown that the Arabidopsis AtSR1 could bind to the CGCG box, a characteristic cis-elements for the SR proteins [12], in the promoter region of EDS1 and suppressed the expression of EDS1 [20], which is critical to biosynthesis of SA. Bioinformatics analysis also identified a CGCG box within $1.3 \mathrm{~Kb}$ from the starting codon in the promoter of tomato SIEDS1 gene [30]. It is thus possible that SISR1 and perhaps SISR3Ll regulate negatively the expression of SIEDS1 through binding to the CGCG box as the expression 
level of SIEDS1 was upregulated when either SISR1 or SISR3L was silenced (Figure 6C). Furthermore, the expression of SIJAZ1, known to be associated with the JA-mediated signaling pathway [39], was downregulated in the SISR1- and SlSR3L-silenced plants (Figure 6C), suggesting a negative impact of SISR1 and SISR3L on the JA-mediated signaling pathway. This is in agreement with the observations that the Arabidopsis AtSR1 is a negative regulator for JA biosynthesis and herbivory tolerance [22,23]. Another possibility that the constitutively activated SA-mediated signaling pathway in the SISR1- and SISR3L-silenced plants antagonistically suppressed the JA-mediated signaling pathway cannot be ruled out because the antagonistic cross-talk between these two signaling pathways is a common phenomenon occurred in regulating defense response against infection by different pathogens [51]. Taken together, these data suggest that both SISR1 and SISR3L negatively regulate basic immunity in tomato through modulating the SAand ET-mediated signaling pathways.

The involvement of the SR family members in abiotic stress was recently investigated using Arabidopsis knockout mutant lines [25,27]. Our VIGS-based functional analyses discovered that silencing of SISR1L resulted in decreased drought stress tolerance (Figures $7 \mathrm{E}$ and $8 \mathrm{~A}$ ). SISR1L is phylogenetically related to the Arabidopsis AtSR2 [29], which was shown to regulate drought stress responses [27]. Thus, it is likely that SISR1L plays an important role in regulation of drought stress tolerance in tomato. The expression of SISR1L was induced significantly by drought stress in detached leaves and in whole plants (Figure 7A and D). The decreased drought tolerance in the SISR1L-silenced plants might be caused by multiple factors including morphological, physiological and molecular changes, which are affected by loss-offunction of SISR1L. Like the stunted primary root in the Arabidopsis $s r 2$ mutant plants [27], the SlSR1L-silenced plants had limited root system and biomass under normal growth condition (Figure $8 \mathrm{C}$ and $\mathrm{D}$ ). Although the exact function of SISR1L in development of the root system in tomato needs to be further investigated, the limited root system in the SISR1L-silenced plants may result in reduced capacity of water uptake. Another fact that affected the water status in the SISR1L-silenced plants was the accelerated rate of water loss, as revealed in the detached leaves (Figure 8B). Furthermore, the expression of SIAREB1 [42], SIDREB [43], SISpUSP [44], SlAREB2 [42] and SGN213276 [40] was suppressed in the SlSR1L-silenced plants (Figure 8E), indicating that SISR1L may regulate the expression of a large set of drought stress-responsive genes. Recent microarray-based analyses of gene expression profiling between the Arabidopsis srl and wild type plants revealed that hormone-mediated signaling such as ABA-mediated signaling plays important roles in
AtSR1-regulated abiotic stress response [18,27]. However, exogenous ABA did not induce the expression of SISR1L in tomato (Figure 7B) and AtSR2 in Arabidopsis [12]. Thus, it is likely that, as a transcription factor, SISR1L acts in a yet-unknown signaling pathway, in which some hormones such as ABA are involved, to regulate drought stress response in tomato.

Our data presented in this study clearly demonstrate that SISR1/SISR3L and SISR1L play important roles in biotic and abiotic stress responses, respectively. However, several questions regarding the mechanism of action of SISR1/SISR3L and SISR1L in biotic and abiotic stress response need to be addressed. Further identification of downstream target genes regulated by SISR1/SISR3L and SISR1L will help to elucidate the molecular mechanisms and the signaling pathways involved in the SISR1/SISR3Lregulated defense response against pathogens and the SISR1L-regulated drought stress response. Of particular, the SISR3L will be a priority for further study because the function of its orthologs in other plants such as the Arabidopsis AtSR3 and AtSR6 [29] has not been defined yet. On the other hand, biochemical studies have shown that the Arabidopsis AtSR2/CAMTA1 [11], rice OsCBT [14] and tomato SISR1 and SISR3L (Figure 9) are functionally transcriptional activators in yeast. However, the Arabidopsis AtSR1 was shown to bind to the CGCG box in the promoters of the EDS1, NDR1 and EIN3 genes and repress their expression $[20,21]$. Thus, the biochemical mechanism regarding how the SR proteins as transcriptional activators repress the expression of the target genes after binding to the CGCG box in the promoters of these genes needs to be further investigated.

\section{Conclusion}

Tomato genome encodes seven SlSR genes and expression of SISR1 and SISR3L was significantly induced by B. cinerea and Pst DC3000. Silencing of either SISR1 or SlSR3L resulted in enhanced resistance to $B$. cinerea and Pst DC3000 and led to constitutive accumulation of $\mathrm{H}_{2} \mathrm{O}_{2}$, elevated expression of defense genes, PTI marker genes and regulatory genes involved in the SA- and ET-mediated signaling pathways. Meanwhile, expression of SISR1L was significantly induced by drought stress and silencing of SISR1L led to decreased drought stress tolerance. These results demonstrate that both SISR1 and SISR3L in the tomato SISR/CAMTA family are negative regulators of defense response against $B$. cinerea and Pst DC3000 while SISR1L is a positive regulator of drought stress tolerance in tomato.

\section{Methods}

\section{Plant growth and treatments}

Tomato (Solanum lycopersicum) cv. Suhong 2003 was used for all experiments. Seeds were scarified on moist 
filter paper in Petri dishes for 2 days and the sprouted seeds were transferred into a mixture of perlite: vermiculite: plant ash (1:6:2). All tomato plants were grown in a growth room at $22-24^{\circ} \mathrm{C}$ with $60 \%$ relative humidity (RH) under a $14 \mathrm{hr}$ light $\left(350 \mu \mathrm{mol} \cdot \mathrm{s}^{-1} \cdot \mathrm{m}^{-2}\right.$ photons $\mathrm{m}^{-2}$ $\left.\sec ^{-1}\right) / 10 \mathrm{hr}$ dark cycle. Two-week-old plants were used for VIGS assays and four-week-old plants were used for other experiments. For analysis of gene expression in response to pathogen infection, whole plant inoculation assays for $B$. cinerea and vacuum infiltrated inoculation assays for Pst DC3000 along with corresponding mockinoculation controls were performed (see below). For analysis of gene expression in drought stress, drought stress was applied to the plants by stopping watering for a period until wilting symptom appeared and normally watered plants were used as controls in the whole plant drought stress assays. Alternatively, fully expanded leaves were detached and subjected to drought stress treatment by placing on lab blench or on water-saturated filter papers in Petri dishes as controls in detached leaf assays. For ABA treatment, a solution of ABA $(100 \mu \mathrm{M})$ or same volume of water as a control was sprayed onto leaf surface of the tomato plants. Leaf samples were collected at indicated time points after treatment or inoculation and stored at $-80^{\circ} \mathrm{C}$ until use.

\section{Plant inoculation and disease assays}

Pst DC3000 was grown overnight in King's B (KB) liquid medium containing rifampicin at $50 \mu \mathrm{g} / \mathrm{ml}$. The bacteria were collected and resuspended in $10 \mathrm{mM} \mathrm{MgCl}$ to $\mathrm{OD}_{600}=0.0002$ for plant inoculation. Four-week-old plants were vacuum infiltrated with suspension of Pst DC3000 or with $\mathrm{MgCl}_{2}$ solution as a mock inoculation control. The inoculated plants were kept in a sealed container to maintain high humidity $(\mathrm{RH}>90 \%)$ and disease progress was observed daily. Leaf samples were collected from at least six Pst DC3000-inoculated or mock-inoculated plants at different time points after inoculation and used for analysis of gene expression and in planta bacterial growth. For measurement of bacterial growth, leaf discs (6 $\mathrm{mm}$ in diameter) were surface sterilized in $70 \%$ ethanol for $10 \mathrm{~s}$, homogenized in $200 \mu \mathrm{l}$ of $10 \mathrm{mM} \mathrm{MgCl}$, diluted in $10 \mathrm{mM}$ $\mathrm{MgCl}_{2}$, and plated on $\mathrm{KB}$ agar plates containing rifampicin at $50 \mu \mathrm{g} / \mathrm{ml}$. The plates were incubated at $28^{\circ} \mathrm{C}$ and the bacterial numbers were counted 3 days after incubation.

B. cinerea was grown on $2 \times \mathrm{V} 8$ agar (36\% V8 juice, $0.2 \% \mathrm{CaCO}_{3}$, and $2 \%$ agar) at $22^{\circ} \mathrm{C}$ and spores were collected and resuspended in $1 \%$ maltose buffer to $2 \times 10^{5}$ spores $/ \mathrm{ml}$ for whole plant inoculation and $1 \times 10^{5}$ spores $/ \mathrm{ml}$ for detached leaf inoculation. Whole plant inoculation and detached leaf inoculation assays were performed according to previously reported procedure
$[52,53]$. In the detached leaf inoculation assays, leaves were detached from at least 10 four-week-old VIGSinfiltrated plants and inoculated by dropping $5 \mu \mathrm{l}$ of spore suspension on leaf surface. In the whole plant inoculation assays, four-week-old plants were inoculated by foliar spraying with spore suspension of $B$. cinerea or with same volume of $1 \%$ maltose buffer as a mock-inoculation control and leaf samples were collected at different time points after inoculation for analysis of gene expression and in planta fungal growth. The inoculated leaves and plants were kept at $22^{\circ} \mathrm{C}$ in sealed containers to retain the moist conditions favorable for disease development. In planta fungal growth was analyzed by the amplification of the transcripts of a B. cinerea Actin gene as a marker [53,54] using a pair of primers BcActin-F and BcActin-R (Additional file 2). Relative fungal growth was expressed as folds of the transcript levels of BcActin vs the transcript levels of a tomato Actin gene.

\section{Construction of vectors and VIGS assays}

Based on the cDNA sequences of all seven SISR genes [29], fragments of 386-462 bp for each SlSR gene (Additional file 3) were amplified with gene-specific primers (Additional file 2) from cDNAs synthesized from total RNA prepared from tomato leaf samples. After cloning and sequencing, these VIGS fragments were cloned into pTRV2 vector [32], yielding pTRV2-SISRs. These pTRV2-SISR constructs were then introduced into Agrobacterium tumefaciens strain GV3101 by electroporation using GENE PULSER II Electroporation System (Bio-Rad Laboratories, Hercules, CA, USA). Agrobacteria carrying pTRV2-GUS (as a negative control), pTRV2-PDS (as a positive control for silencing efficiency examination) or pTRV2-SISRs were cultivated in YEP medium $(10 \mathrm{~g} / \mathrm{l}$ peptone, $10 \mathrm{~g} / \mathrm{l}$ yeast extract, $5 \mathrm{~g} \mathrm{NaCl} / \mathrm{l}, 50 \mu \mathrm{g} / \mathrm{ml}$ rifampicin, $50 \mu \mathrm{g} / \mathrm{ml}$ kanamycin and $25 \mu \mathrm{g} / \mathrm{ml}$ gentamicin) for $36 \mathrm{hr}$ with continuous shaking in a $28^{\circ} \mathrm{C}$ incubator. Cells were centrifuged and resuspended in infiltration buffer $(10 \mathrm{mM} \mathrm{MgCl}$, $150 \mu \mathrm{M}$ acetosyringone, MES, pH5.7). The agrobacteria carrying pTRV2-GUS, pTRV2-PDS or pTRV2-SISR were mixed with the agrobacteria carrying pTRV1 in a ratio of $1: 1$ and maintained at $\mathrm{OD}_{600}=1.5$ for $3 \mathrm{hr}$ at room temperature. The mixed agrobacteria suspension was infiltrated into the abaxial surface of the 2-week-old seedlings using $1 \mathrm{~mL}$ needleless syringes. Efficiency of the silencing procedure was evaluated based on the appearance of bleaching phenotype in the pTRV2PDS-infiltrated plants [32]. When more than $90 \%$ of the pTRV2-PDS-infiltrated plants showed bleaching phenotype, the pTRV2-GUS- or pTRV2-SISR-infiltrated plants in an independent experiment with same VIGS procedure were used for further study. Leaf samples 
were collected at 2 weeks after VIGS infiltration and used for analysis of the silencing efficiency by qRTPCR.

\section{Subcellular localization}

The coding sequences of SISR 1 and SISR3L were PCR amplified using pairs of gene-specific primers SISR13F/SISR1-3R and SISR3L-3F/SISR3L-3R, respectively (Additional file 2). After confirmation by sequencing, the coding regions of the SISR 1 and SISR3L genes were cloned into pFGC-Egfp. The recombinant plasmids pFGCEgfp-SlSR1, pFGC-Egfp-SISR3L and pFGC-Egfp were transformed into A. tumefacies strain GV3101 and the transformed agrobacteria were infiltrated individually into leaves of four-week-old $N$. benthamiana plants using 1 -ml needless syringes. These agroinfiltrated plants were allowed to grow in a growth chamber at $25^{\circ} \mathrm{C}$ for $48 \mathrm{hr}$, and the GFP fluorescence was examined under a Leica TCS SP5 laser confocal microscope with excitation wavelength of $488 \mathrm{~nm}$.

\section{Transcription activation assay in yeast}

The coding sequences of SISR1 and SlSR3L were amplified using gene-specific primers (Additional file 2) and cloned into pBD-GAL4Cam vector to yield pBD-SISR1 and $\mathrm{pBD}-\mathrm{SISR} 3 \mathrm{~L}$. These two recombinant plasmids and the $\mathrm{pBD}$ empty vector (as a negative control) were transformed into yeast strain AH109. The transformed yeasts were confirmed by colony PCR and then cultivated on the $\mathrm{SD} /-\operatorname{Trp}$ and $\mathrm{SD} /-\mathrm{Trp} /-$ His medium for 3 days at $28^{\circ} \mathrm{C}$, followed by addition of $\mathrm{x}$ - $\alpha$-gal (5-bromo-4chloro-3-indolyl- $\alpha$-D-galactopyranoside). Transactivation activity of the fused proteins was evaluated according to the growth situation and production of blue pigments after the addition of $\mathrm{x}$ - $\alpha$-gal of the transformed yeast cells on the $\mathrm{SD} /-\mathrm{Trp} /-$ His medium.

\section{Detection of $\mathrm{H}_{2} \mathrm{O}_{2}$ accumulation}

Detection of in situ $\mathrm{H}_{2} \mathrm{O}_{2}$ was carried out using DAB staining method as described previously [55]. Leaves were collected from pTRV2-SISR1- or pTRV2-SISR3Linfiltrated and pTRV2-GUS-infiltrated plants and dipped into DAB solution $(1 \mathrm{mg} / \mathrm{ml}, \mathrm{pH} 3.8)$ and incubated for 8 $\mathrm{hr}$ in dark at room temperature. Thereafter, leaves were placed into acetic acid/glycerol/ethanol (1:1:1, vol/vol/ vol) and boiled for $5 \mathrm{~min}$ in a water bath. After several changes of the solution, then leaves were maintained in $60 \%$ glycerol and accumulation of $\mathrm{H}_{2} \mathrm{O}_{2}$ in leaves was photographed using a digital camera.

\section{Drought tolerance assays}

At least 10 individual pTRV2-SlSRs- or pTRV2-GUSinfiltrated plants were used in each experiment. Drought stress was applied to the tomato plants by stopping watering for a certain period of 10-12 days or until the wilting symptoms were obvious. To assess the ratio of water loss, fully expanded leaves from 6 individual pTRV2-SISR1L- or pTRV2-GUS-infiltrated plants were detached and placed on the bench top. The weights of the leaves were recorded at different time points after detachment and the average water loss ratio was calculated by comparing with the initial weights. Roots from 6 individual pTRV2-SISR1L- or pTRV2-GUS-infiltrated plants were cut, cleaned and dried in $70^{\circ} \mathrm{C}$ oven for $24 \mathrm{hr}$ and the final weight was calculated and compared. Leaf samples were collected from the pTRV2-SISRs- or pTRV2-GUS-infiltrated plants at $0 \mathrm{hr}$ (as unstressed control) and 7 days after stopping watering and were subjected to analysis of gene expression.

\section{qRT-PCR analysis of gene expression}

Total RNA was extracted from frozen leaf samples using TRIzol reagent (Invitrogen, Shanghai, China) and treated with RNase-free DNase (TaKaRa, Dalian, China) to erase any genomic DNA in the RNA samples. First-strand cDNA was synthesized from $0.6 \mu \mathrm{g}$ total RNA using AMV reverse transcriptase (TaKaRa, Dalian, China) according to the manufacturer's recommendations. Each qRT-PCR reaction contained $12.5 \mu \mathrm{l}$ SYBR Premix Ex Taq (TaKaRa, Dalian, China), $0.1 \mu \mathrm{g}$ cDNA and $7.5 \mathrm{pmol}$ of each gene-specific primer (Additional file 2) in a final volume of $25 \mu \mathrm{l}$, and run on three independent biological replicates. The qRT-PCR was performed in a CFX96 realtime PCR detection system (BioRad, Hercules, CA, USA) and relative expression levels were calculated using the $2^{-\Delta \Delta C T}$ method. The expression level of a tomato actin gene was used as an internal control to normalize the expression data for the target genes. Relative expression levels of the target genes were shown as folds of the expression level of the actin gene or as folds of the expression levels in treated plants/control plants.

\section{Statistical analysis}

All experiments were repeated independently three times and data were collected from experiments on three biological samples. All data obtained were subjected to statistical analysis according to the Student's $t$-test and the probability values of $p<0.05$ were considered as significant difference.

\section{Accession numbers for SISRs}

The tomato SlSR gene sequences used in this study were retrieved from GenBank under the following accession numbers: SlSR1, GU170838; SlSR1L, JN558810; SlSR2, JN566047; SlSR2L, JN566048; SlSR3, JN566049; SlSR3L, JN566051; SlSR4, JN566050. These SlSR gene sequences were deposited by Yang et al. [29]. 


\section{Availability of supporting data}

The sequences of SISR proteins and sequences of VIGS fragments for SlSR genes used in this study are included in Additional files 1 and 3.

\section{Additional files}

Additional file 1: Alignment of tomato SISR protein sequences and positions of the VIGS fragments in the SISR proteins. The GenBank accession numbers for SISR proteins are as follows: SISR1, ADK47999; SISR1L, AEX31181; SISR2, AEX07774; SISR2L, AEX07775; SISR3, AEX07776; SISR3L, AEX07778; SISR4, AEX07777. These SISR protein sequences were deposited by Yang et al. [29]. Regions selected for the VIGS fragments of each of SISR genes were underlined with red lines.

Additional file 2: Table S1. Primers used in this study. Additional file 3: Sequences of the VIGS fragment for SISR genes.

\section{Competing interests}

The authors declare that they have no competing interests.

\section{Authors' contributions}

$X L, L H, Y Z, Z O, Y H, H Z$ and $D L$ carried out the experiments. $X L$ and $F S$ designed the experiments. FS and $X \mathrm{~L}$ wrote the paper. All authors read and approved the final manuscript.

\section{Acknowledgements}

This work was supported by the National High-Tech R \& D Program (No. 2012AA101504 and No. 2012AA101505), the National Basic Research Program of China (2009CB119005) and the Research Fund for the Doctoral Program of Higher Education of China (20120101110070).

Received: 2 August 2014 Accepted: 15 October 2014 Published online: 28 October 2014

\section{References}

1. Kudla J, Batistic O, Hashimoto K: Calcium signals: the lead currency of plant information processing. Plant Cell 2010, 22(3):541-563.

2. McAinsh MR, Pittman JK: Shaping the calcium signature. New Phytol 2009, 181(2):275-294.

3. Plieth C: Signal percolation through plants and the shape of the calcium signature. Plant Signal Behav 2010, 5(4):379-385.

4. Ma W, Berkowitz GA: The grateful dead: calcium and cell death in plant innate immunity. Cell Microbiol 2007, 9(11):2571-2585.

5. Reddy ASN, Ali GS, Celesnik H, Day IS: Coping with stresses: roles of calcium- and calcium/calmodulin-regulated gene expression. Plant Cell 2011, 23(6):2010-2032

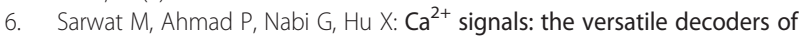
environmental cues. Crit Rev Biotechnol 2013, 33(1):97-109.

7. Poovaiah BW, Du LQ, Wang HZ, Yang TB: Recent advances in calcium/ calmodulin-mediated signaling with an emphasis on plant-microbe interactions. Plant Physiol 2013, 163(2):531-542.

8. Zielinski RE: Calmodulin and calmodulin-binding proteins in plants. Annu Rev Plant Biol 1998, 49:697-725.

9. Bouche N, Yellin A, Snedden WA, Fromm H: Plant-specific calmodulin-binding proteins. Annu Rev Plant Biol 2005, 56:435-466.

10. Luan S, Kudla J, Rodriguez-Concepcion M, Yalovsky S, Gruissem W: Calmodulins and calcineurin Bac like proteins: calcium sensors for specific signal response coupling in plants. Plant Cell 2002, 14(Suppl):S389-S400.

11. Bouche N, Scharlat A, Snedden W, Bouchez D, Fromm H: A novel family of calmodulin-binding transcription activators in multicellular organisms. J Biol Chem 2002, 277(24):21851-21861.

12. Yang TB, Poovaiah BW: A calmodulin-binding/CGCG box DNA-binding protein family involved in multiple signaling pathways in plants. $J$ Biol Chem 2002, 277(47):45049-45058.
13. Finkler A, Ashery-Padan R, Fromm H: CAMTAs: calmodulin-binding transcription activators from plants to human. FEBS Lett 2007, 581(21):3893-3898.

14. Choi MS, Kim MC, Yoo JH, Moon BC, Koo SC, Park BO, Lee JH, Koo YD, Han HJ, Lee SY, Chung WS, Lim CO, Cho MJ: Isolation of a calmodulin-binding transcription factor from rice (Oryza sativa L.). J Biol Chem 2005, 280(49):40820-40831.

15. Reddy ASN, Reddy VS, Golovkin M: A calmodulin binding protein from Arabidopsis is induced by ethylene and contains a DNA-binding motif. Biochem Biophys Res Commun 2000, 279(3):762-769.

16. Yang TB, Poovaiah BW: An early ethylene up-regulated gene encoding a calmodulin-binding protein involved in plant senescence and death. J Biol Chem 2000, 275(49):38467-38473.

17. Koo SC, Choi MS, Chun HJ, Shin DB, Park BS, Kim YH, Park HM, Seo HS, Song JT, Kang KY, Yun DJ, Chung WS, Cho MJ, Kim MC: The calmodulin-binding transcription factor OsCBT suppresses defense responses to pathogens in rice. Mol Cells 2009, 27(5):563-570.

18. Galon Y, Aloni R, Nachmias D, Snir O, Feldmesser E, Scrase-Field S, Boyce JM, Bouche N, Knight MR, Fromm H: Calmodulin-binding transcription activator 1 mediates auxin signaling and responds to stresses in Arabidopsis. Planta 2010, 232(1):165-178.

19. Galon $Y$, Nave R, Boyce JM, Nachmias D, Knight MR, Fromm H: Calmodulin-binding transcription activator (CAMTA) 3 mediates biotic defense responses in Arabidopsis. FEBS Lett 2008, 582(6):943-948.

20. Du LQ, Ali GS, Simons KA, Hou JG, Yang TB, Reddy ASN, Poovaiah BW: $\mathrm{Ca}^{2+} /$ calmodulin regulates salicylic-acid-mediated plant immunity. Nature 2009, 457(7233):1154-1158.

21. Nie HZ, Zhao CZ, Wu GH, Wu YY, Chen YF, Tang DZ: SR1, a calmodulin-binding transcription factor, modulates plant defense and ethylene-induced senescence by directly regulating NDR1 and EIN3. Plant Physiol 2012, 158(4):1847-1859.

22. Laluk K, Prasad KVSK, Savchenko T, Celesnik H, Dehesh K, Levy M, Mitchell-Olds T, Reddy ASN: The calmodulin-binding transcription factor signal responsive1 is a novel regulator of glucosinolate metabolism and herbivory tolerance in Arabidopsis. Plant Cell Physiol 2012, 53(12):2008-2015.

23. Qiu Y, Xi J, Du L, Suttle JC, Poovaiah BW: Coupling calcium/calmodulinmediated signaling and herbivore-induced plant response through calmodulin-binding transcription factor AtSR1/CAMTA3. Plant Mol Biol 2012, 79(1-2):89-99.

24. Jing B, Xu S, Xu M, Li Y, Li S, Ding J, Zhang Y: Brush and spray: a high throughput systemic acquired resistance assay suitable for large scale genetic screening. Plant Physiol 2011, 157(3):973-980.

25. Doherty CJ, Buskirk HAV, Myers SJ, Thomashow MF: Roles for Arabidopsis CAMTA transcription factors in cold-regulated gene expression and freezing tolerance. Plant Cell 2009, 21(3):972-984.

26. Kim YS, Park SC, Gilmour SJ, Thomashow MF: Roles of CAMTA transcription factors and salicylic acid in configuring the low-temperature transcriptome and freezing tolerance of Arabidopsis. Plant J 2013, 75(3):364-376.

27. Pandey N, Ranjan A, Pant P, Tripathi RK, Ateek F, Pandey HP, Patre UV, Sawant SV: CAMTA1 regulates drought responses in Arabidopsis thaliana. BMC Genomics 2013, 14:216

28. Zegzouti H, Jones B, Frasse P, Marty C, Maitre B, Latche AA, Pech JC, Bouzayen $M$ : Ethylene-regulated gene expression in tomato fruit: characterization of novel ethylene-responsive and ripening-related genes isolated by differential display. Plant J 1999, 18(6):589-600.

29. Yang TB, Peng $H$, Whitaker BD, Conway WS: Characterization of a calcium/calmodulin regulated SR/CAMTA gene family during tomato fruit development and ripening. BMC Plant Biol 2012, 12:19.

30. Yang TB, Peng $H$, Whitaker BD, Jurick WM: Differential expression of calcium/calmodulin-regulated SISRs in response to abiotic and biotic stresses in tomato fruit. Physiol Plant 2013, 148(3):445-455.

31. Kawazu K, Mochizuki A, Sato Y, Sugeno W, Murata M, Seo S, Mitsuhara I: Different expression profiles of jasmonic acid and salicylic acid inducible genes in the tomato plant against herbivores with various feeding modes. Arthropod Plant Interact 2012, 6:221-230.

32. Liu Y, Schiff M, Dinesh-Kumar SP: Virus-induced gene silencing in tomato. Plant J 2002, 31(6):777-786

33. Marino D, Dunand C, Puppo A, Pauly N: A burst of plant NADPH oxidases. Trends Plant Sci 2012, 17(1):9-15. 
34. Kim JG, Li X, Roden JA, Taylor KW, Aakre CD, Su B, Lalonde S, Kirik A, Chen Y, Baranage G, McLane H, Martin GB, Mudgett MB: Xanthomonas T3S effector XopN suppresses PAMP-triggered immunity and interacts with a tomato atypical receptor-like kinase and TFT1. Plant Cell 2009, 21(4):1305-1323.

35. Nguyen HP, Chakravarthy S, Velásquez AC, McLane HL, Zeng L, Nakayashiki H, Park DH, Collmer A, Martin GB: Methods to study PAMP-triggered immunity using tomato and Nicotiana benthamiana. Mol Plant-Microbe Interact 2010, 23(8):991-999.

36. Ekengren SK, Liu Y, Schiff M, Dinesh-Kumar SP, Martin GB: Two MAPK cascades, NPR1, and TGA transcription factors play a role in Pto-mediated disease resistance in tomato. Plant J 2003, 36(6):905-917.

37. Kamiyoshihara Y, Tieman DM, Huber DJ, Klee HJ: Ligand-induced alterations in the phosphorylation state of ethylene receptors in tomato fruit. Plant Physio/ 2012, 160(1):488-497.

38. Di Matteo A, Ruggieri V, Sacco A, Rigana MM, Carriero F, Bolger A, Fernie $A R$, Frusciante $L$, Barone $A$ : Identification of candidate genes for phenolics accumulation in tomato fruit. Plant Sci 2013, 205-206:87-96.

39. Pauwels $L$, Goossens $A$ : The JAZ proteins: a crucial interface in the jasmonate signaling cascade. Plant Cell 2011, 23(9):3089-3100.

40. Gong PJ, Zhang J, Li H, Yang C, Zhang C, Zhang X, Khurram Z, Zhang Y, Wang T, Fei Z, Ye Z: Transcriptional profiles of drought-responsive genes in modulating transcription signal transduction, and biochemical pathways in tomato. J Exp Bot 2010, 61(13):3563-3575.

41. Wasilewska A, Vlad F, Sirichandra C, Redko Y, Jammes F, Valon C, Freid it Frey N, Leung J: An update on abscisic acid signaling in plants and more.... Mol Plant 2008, 1(2):198-217.

42. Orellana S, Yanez M, Espinoza A, Verdugo I, Gonzalez E, RuizLara S, Casaretto JA: The transcription factor SIAREB1 confers drought, salt stress tolerance and regulates biotic and abiotic stress-related genes in tomato. Plant Cell Environ 2010, 33(12):2191-2208

43. Li J, Sima W, Ouyang B, Wang TT, Ziaf K, Luo ZD, Liu LF, Li HX, Chen ML, Huang $Y Q$, Feng $Y Q$, Hao $Y H$, Ye ZB: Tomato SIDREB gene restricts leaf expansion and internode elongation by downregulating key genes for gibberellin biosynthesis. J Exp Bot 2012, 63(18):6407-6420.

44. Loukehaich R, Wang T, Ouyang B, Ziaf K, Li H, Zhang J, Lu Y, Ye Z: SpUSP, an annexin-interacting universal stress protein, enhances drought tolerance in tomato. J Exp Bot 2012, 63(15):5593-5606.

45. Guo $Y$, Huang $C$, Xie $Y$, Song F, Zhou X: A tomato glutaredoxin gene SIGRX1 regulates plant responses to oxidative, drought and salt stresses. Planta 2010, 232(6):1499-1509.

46. Sagi M, Davydov O, Orazova S, Yesbergenova Z, Ophir R, Stratmann JW, Fluhr R: Plant respiratory burst oxidase homologs impinge on wound responsiveness and development in Lycopersicon esculentum. Plant Cell 2004, 16(3):616-628.

47. Guan LM, Scandalios JG: Hydrogen peroxide-mediated catalase gene expression in response to wounding. Free Radical Bio Med 2000, 28(8):1182-1190.

48. Guan LM, Zhao J, Scandalios JG: Cis-elements and trans-factors that regulate expression of the maize Cat 1 antioxidant gene in response to ABA and osmotic stress: $\mathrm{H}_{2} \mathrm{O}_{2}$ is the likely intermediary signaling molecule for the response. Plant J 2000, 22(2):87-95.

49. Mengiste T: Plant immunity to necrotrophs. Annu Rev Phytopathol 2012 50:267-294.

50. Jones JD, Dangl JL: The plant immune system. Nature 2006, 444(7117):323-329.

51. Glazebrook J: Contrasting mechanisms of defense against biotrophic and necrotrophic pathogens. Annu Rev Phytopathol 2005, 43:205-227.

52. AbuQamar S, Chai MF, Luo H, Song F, Mengiste T: Tomato protein kinase $1 \mathrm{~b}$ mediates signaling of plant responses to necrotrophic fungi and insect herbivory. Plant Cell 2008, 20(7):1964-1983.

53. Li X, Zhang Y, Huang L, Ouyang Z, Hong Y, Zhang H, Li D, Song F: Tomato SIMKK2 and SIMKK4 contribute to disease resistance against Botrytis cinerea. BMC Plant Biol 2014, 14:166.
54. Benito EP, ten Have A, van't Klooster JW, van Kan JAL: Fungal and plant gene expression during synchronized infection of tomato leaves by Botrytis cinerea. Eur J Plant Pathol 1998, 104(2):207-220.

55. Thordal-Christensen H, Zhang Z, Wei Y, Collinge DB: Subcellular localization of $\mathrm{H}_{2} \mathrm{O}_{2}$ in plants. $\mathrm{H}_{2} \mathrm{O}_{2}$ accumulation in papillae and hypersensitive response during the barley-powdery mildew interaction. Plant J 1997, 11(6):1187-1194

doi:10.1186/s12870-014-0286-3

Cite this article as: Li et al:: Tomato SR/CAMTA transcription factors SISR1 and SISR3L negatively regulate disease resistance response and SISR1L positively modulates drought stress tolerance. BMC Plant Biology 2014 14:286.

\section{Submit your next manuscript to BioMed Central and take full advantage of:}

- Convenient online submission

- Thorough peer review

- No space constraints or color figure charges

- Immediate publication on acceptance

- Inclusion in PubMed, CAS, Scopus and Google Scholar

- Research which is freely available for redistribution 